MATHEMATICS OF COMPUTATION

Volume 71, Number 238, Pages 605-632

S 0025-5718(01)01408-9

Article electronically published on December 5, 2001

\title{
ANALYZING THE STABILITY BEHAVIOUR OF SOLUTIONS AND THEIR APPROXIMATIONS IN CASE OF INDEX-2 DIFFERENTIAL-ALGEBRAIC SYSTEMS
}

\author{
ROSWITHA MÄRZ AND ANTONIO R. RODRÍGUEZ-SANTIESTEBAN
}

\begin{abstract}
When integrating regular ordinary differential equations numerically, one tries to match carefully the dynamics of the numerical algorithm with the dynamical behaviour of the true solution. The present paper deals with linear index-2 differential-algebraic systems. It is shown how knowledge pertaining to (numerical) regular ordinary differential equations applies provided a certain subspace which is closely related to the tangent space of the constraint manifold remains invariant.
\end{abstract}

\section{INTRODUCTION}

Usually, lower-index differential-algebraic equations (DAEs)

$$
f\left(x^{\prime}(t), x(t), t\right)=0
$$

are integrated by numerical algorithms that have been developed originally for regular ordinary differential equations (ODEs). There are numerous papers justifying this by convergence proofs, order results, etc. (cf. 1], 2], [3]). In particular, the backward differentiation formula (BDF)

$$
f\left(\frac{1}{h} \sum_{j=0}^{k} \alpha_{j} x_{n-j}, x_{n}, t_{n}\right)=0,
$$

and also implicit Runge-Kutta methods (IRK)

$$
\begin{gathered}
x_{n}=x_{n-1}+h \sum_{j=1}^{s} \beta_{j} X_{n j}^{\prime}, \\
f\left(X_{n i}^{\prime}, x_{n-1}+h \sum_{j=1}^{s} \alpha_{i j} X_{n j}^{\prime}, t_{n-1}+c_{i} h\right)=0, \quad i=1, \ldots, s,
\end{gathered}
$$

are used in applications with great success. Step by step, each method provides numerical approximations $x_{n}$ of the true solution values $x\left(t_{n}\right), t_{n}=t_{n-1}+h$.

The convergence results mentioned above concern the behaviour of the global error on a compact interval, say $\left[t_{0}, T\right]$, as the stepsize of the discretization $t_{0}<$ $t_{1}<\cdots<t_{n}=T$ tends to zero.

Received by the editor August 25, 1999.

2000 Mathematics Subject Classification. Primary 65L20; Secondary 34D05.

Key words and phrases. Differential-algebraic equations, numerical stability, logarithmic norms, contractivity. 
However, what do we know about the error $x\left(t_{n}\right)-x_{n}$ if the stepsize $h$ is constant and $n \rightarrow \infty$, that is, $t_{n} \rightarrow \infty$ ? When integrating regular ODEs numerically, one tries to carefully match the dynamics of the numerical algorithm with the dynamical behaviour of the true solution. How could this be done in case of DAEs?

Classical linear stability theory is concerned with the analysis of approximating the so-called scalar linear test equation

$$
z^{\prime}(t)=\lambda z(t)
$$

Basic notions like the region of absolute stability, error growth function, etc., rely on (1.4) and apply, via similarity transforms, to constant coefficient regular systems

$$
x^{\prime}(t)-W x(t)=0 .
$$

Considering DAEs, the corresponding constant coefficient system

$$
A x^{\prime}(t)+B x(t)=0
$$

has a singular leading coefficient matrix $A$, but a regular matrix pencil $\{A, B\}$, i.e., $\operatorname{det}(\lambda A+B) \not \equiv 0$. There are nonsingular matrices $E, F$ that transform (1.6) into its so-called Kronecker normal form

$$
\left(\begin{array}{ll}
I & \\
& J
\end{array}\right) \tilde{x}^{\prime}(t)+\left(\begin{array}{ll}
-W & \\
& I
\end{array}\right) \tilde{x}(t)=0,
$$

where $E A F=\left(\begin{array}{cc}I & \\ & J\end{array}\right), E B F=\left(\begin{array}{cc}-W & \\ & I\end{array}\right), \tilde{x}=F^{-1} x$, and $J$ is a nilpotent Jordan block matrix, ind $(J)=\operatorname{ind}\{A, B\}$. Obviously, the solution of the homogeneous equation (1.6) consists of its "dynamical part" only, i.e.,

$$
x(t)=F \tilde{x}(t)=F\left(\begin{array}{c}
\tilde{u}(t) \\
0
\end{array}\right),
$$

where $\tilde{u}(t)$ solves the regular ODE $\tilde{u}^{\prime}(t)=W \tilde{u}(t)$. On the other hand, we may apply the same transformations $E, F$ to decouple the BDF applied to (1.6), that is,

$$
A \frac{1}{h} \sum_{j=0}^{k} \alpha_{j} x_{n-j}+B x_{n}=0
$$

to

$$
\left(\begin{array}{ll}
I & \\
& J
\end{array}\right) \frac{1}{h} \sum_{j=0}^{k} \alpha_{j} \tilde{x}_{n-j}+\left(\begin{array}{cc}
-W & \\
& I
\end{array}\right) \tilde{x}_{n}=0
$$

Starting with consistent values $x_{0}, \cdots, x_{k-1}\left(\right.$ such that $F^{-1} x_{j}=\left(\begin{array}{c}\tilde{u}_{j} \\ 0\end{array}\right), j=$ $0, \cdots, k)$, we obtain

$$
x_{n}=F \tilde{x}_{n}=F\left(\begin{array}{c}
\tilde{u}_{n} \\
0
\end{array}\right),
$$

where $\tilde{u}_{n}$ is given by the BDF applied to the regular ODE $\tilde{u}^{\prime}(t)=W \tilde{u}(t)$, i.e.,

$$
\frac{1}{h} \sum_{j=0}^{k} \alpha_{j} \tilde{u}_{n-j}=W \tilde{u}_{n}
$$

In the same way we may proceed with Runge-Kutta methods. Obviously, the rich world of classical stability theory applies to constant coefficient DAEs (1.6) via transformation into Kronecker normal form plus similarity transform. 
However, unfortunately, the homogeneous coefficient DAE (1.6) does not play as a prominent role in the DAE analysis as equation (1.5) does in the regular ODEtheory. DAEs represent a much more complex class of problems. The constant coefficient case (1.6) is only a very poor model, which does not reflect important geometric features of DAEs at all.

Positive results concerning long term integrations of index-1 DAEs are reported already in [4]. If the leading partial Jacobian $f_{x^{\prime}}^{\prime}\left(x^{\prime}, x, t\right)$ in (1.1) is supposed to have an invariant nullspace, then things work well. However it is stressed that varying subspaces may have a derogatory effect.

In [5], the following small example was discussed to show the bad effect of a rotating nullspace. The linear index-1 DAE

$$
\left(\begin{array}{cc}
\delta-1 & \delta t \\
0 & 0
\end{array}\right) x^{\prime}(t)+\mu\left(\begin{array}{cc}
\delta-1 & \delta t \\
\delta-1 & \delta t-1
\end{array}\right) x(t)=0
$$

with real parameters $\mu \neq 0, \delta \neq 1$ has the solutions

$$
\begin{aligned}
& x_{1}(t)=(\delta-1)^{-1}(1-\delta t) x_{2}(t), \\
& x_{2}(t)=\exp ((\delta-\mu) t) x_{2}(0) .
\end{aligned}
$$

The nullspace of the leading coefficient matrix is

$$
N(t):=\left\{z \in \mathbb{R}^{2}:(\delta-1) z_{1}+\delta t z_{2}=0\right\} .
$$

For $\delta \neq 0$, this nullspace varies with $t$. Applying the backward Euler method to (1.8) yields

$$
\begin{aligned}
& x_{n, 1}=(\delta-1)^{-1}\left(1-\delta t_{n}\right) x_{n, 2}, \\
& x_{n, 2}=\frac{1+h \delta}{1+h \mu} x_{n-1,2} .
\end{aligned}
$$

Obviously, we have that $x_{n} \rightarrow 0(n \rightarrow \infty)$ if and only if $|1+h \delta|<|1+h \mu|$. For $h \mu=-1$ the method is not feasible at all. Further, there is a large region in the $(h \delta, h \mu)$-plane where the true solution $x\left(t_{n}\right)$ vanishes asymptotically, but the numerical approximation grows unboundedly. Note that the spectrum of the pencil $\{A(t), B(t)\}$ is time-invariant, namely

$$
\sigma(A(t), B(t)):=\{\lambda \in \mathbb{C}: \operatorname{det}(\lambda A(t)+B(t))=0\}=\{-\mu\} .
$$

If we put $\delta=0$, we obtain again a constant coefficient DAE, and everything is as fine as expected.

One could think that a wrong asymptotic behaviour is always due to rotations of the nullspace of the leading partial Jacobian $f_{x^{\prime}}^{\prime}\left(x^{\prime}, x, t\right)$ in (1.1). Since this matrix (fortunately!) has a constant nullspace or is constant itself in most applications, we can perhaps consider equation (1.8) to be just an academic example. However, for higher index DAEs, similar phenomena of a "wrong numerical dynamical behaviour" may arise even in the case of a constant leading coefficient matrix. Such phenomena are discussed in [6], where linear index-2 DAEs are considered.

Roughly speaking, in [6] it is shown that the numerical approximations fit the dynamical behaviour of the true solution well, supposing that two further characteristic subspaces $N_{1}(t)$ and $S_{1}(t)$ do not vary with $t$.

The aim of the present paper is to show that the same invariance condition for $S_{1}(t)$ alone will do. This weaker condition ensures also an appropriate reflection of the exponential decay. Since e.g., in circuit simulation $S_{1}(t)$ is often invariant (cf. [7]), our new approach is of particular interest for those applications. 
This paper is organized as follows. In Section 2 the related analytical background of the index-2 case is given. Useful decoupling and reduction techniques are provided. We figure out the close relationship between the subspace $S_{1}(t)$ and the tangent space of the constraint manifold, which now also includes hidden constraints. As a by-product, Theorem 2.2 offers a new solvability result with respect to weaker smoothness demands. Further, contractivity is now discussed under those weaker smoothness conditions.

In Section 3 the same decoupling and reduction techniques are applied to BDF and IRK methods, to prove that the time invariance of the subspace $S_{1}(t)$ alone will in fact do.

Finally we demonstrate, by two characteristic examples that are actually in Hessenberg form, how a rotating subspace $S_{1}(t)$ effects the dynamic reflection.

\section{ANALYSIS OF LINEAR INDEX-2 DAE'S}

2.1. Fundamentals. Consider the linear equation

$$
A(t) x^{\prime}(t)+B(t) x(t)=q(t), t \in J:=\left[t_{0}, \infty\right),
$$

where the coefficients are continuous and the matrix $A(t) \in L\left(\mathbb{R}^{m}\right)$ is singular for all $t \in J$, but has constant rank $r$. Introduce the basic subspaces

$$
\begin{aligned}
N(t) & :=\operatorname{ker} A(t), \\
S(t) & :=\left\{z \in \mathbb{R}^{m}: B(t) z \in \operatorname{im} A(t)\right\} .
\end{aligned}
$$

Obviously, each solution of the homogeneous equation satisfies $x(t) \in S(t), t \in J$.

In the following, we assume $N(t)$ to be spanned by $m-r$ continuously differentiable base functions. Then, there is a $C^{1}$ matrix function $Q: J \rightarrow L\left(\mathbb{R}^{m}\right)$ that projects $\mathbb{R}^{m}$ pointwise onto $N(t)$, i.e., $Q(t)^{2}=Q(t)$, im $Q(t)=N(t), t \in J$.

In what follows, $Q$ denotes such a $C^{1}$ projector function onto $N$, and $P:=I-Q$. Recall (e.g., [2]) the equation

$$
A(t)\left\{(P x)^{\prime}(t)-P^{\prime}(t) x(t)\right\}+B(t) x(t)=q(t), \quad t \in J,
$$

to be the precise formulation of 2.1). We are looking for continuous functions $x(\cdot): J \rightarrow \mathbb{R}^{m}$ that have continuously differentiable parts $(P x)(\cdot)$, and that satisfy (2.2) pointwise. Denote this function space by

$$
C_{N}^{1}:=\left\{y \in C\left(J, \mathbb{R}^{m}\right): P y \in C^{1}\left(J, \mathbb{R}^{m}\right)\right\} .
$$

Next, we introduce further subspaces which are relevant for index-2 DAEs [2], namely, with

$$
\begin{aligned}
& A_{1}(t):=A(t)+\left(B(t)-A(t) P^{\prime}(t)\right) Q(t), \quad t \in J, \\
& N_{1}(t):=\operatorname{ker} A_{1}(t), \\
& S_{1}(t):=\left\{z \in \mathbb{R}^{m}: B(t) P(t) z \in \operatorname{im} A_{1}(t)\right\} .
\end{aligned}
$$

Definition. The DAE 2.1) is said to be index-2 tractable if $\operatorname{dim}(N(t) \cap S(t))=$ $\nu>0, \nu$ constant, $N_{1}(t) \cap S_{1}(t)=\{0\}, \quad t \in J$.

Recall that index-2 tractability generalizes the case of Kronecker index 2. Let $Q_{1}(t)$ denote the projector onto $N_{1}(t)$ along $S_{1}(t), t \in J$.

Due to 4], Lemma A.13, the matrix

$$
G_{2}(t):=A_{1}(t)+B(t) P(t) Q_{1}(t)
$$


remains nonsingular now, and $Q_{1}(t)=Q_{1}(t) G_{2}(t)^{-1} B(t) P(t)$ on $J$. Since we may represent

$$
\text { ker } A_{1}(t)=\left(I-P(t) A(t)^{+}\left(B(t)-A(t) P^{\prime}(t)\right) Q(t)\right)(N(t) \cap S(t)),
$$

we know $N_{1}(t)$ to have the same constant dimension $\nu$ as $N(t) \cap S(t)$.

In the following, we drop the argument $t$ unless required for clarity. By straightforward calculations we prove the relations

$$
G_{2}^{-1} A=P_{1} P, \quad G_{2}^{-1} B=G_{2}^{-1} B P P_{1}+Q_{1}+Q+P_{1} P P^{\prime} Q .
$$

Hence, scaling equation (2.1) by $G_{2}^{-1}$ leads to

$$
P_{1} P\left((P x)^{\prime}-P^{\prime} x\right)+G_{2}^{-1} B P P_{1} x+Q_{1} x+Q x+P_{1} P P^{\prime} Q x=G_{2}^{-1} q .
$$

Multiplying (2.3) by $P P_{1}, Q P_{1}$ and $Q_{1}$, respectively, and carrying out simple computations, we obtain the decoupled system

$$
\begin{aligned}
P P_{1}(P x)^{\prime}+P P_{1} G_{2}^{-1} B P P_{1} x & =P P_{1} G_{2}^{-1} q, \\
-Q Q_{1}(P x)^{\prime}+Q P_{1} G_{2}^{-1} B P P_{1} x+Q x & =Q P_{1} G_{2}^{-1} q, \\
Q_{1} x & =Q_{1} G_{2}^{-1} q .
\end{aligned}
$$

The system (2.4), (2.5), (2.6) provides the basic idea of what the solutions of the DAE (2.1) look like in case of $P P_{1}, P Q_{1}$ and $P Q_{1} G_{2}^{-1} q$ being $C^{1}$. Then (2.4), (2.5) may be rewritten as

$$
\begin{gathered}
\left(P P_{1} x\right)^{\prime}-\left(P P_{1}\right)^{\prime} P P_{1} x+P P_{1} G_{2}^{-1} B P P_{1} x=P P_{1} G_{2}^{-1} q+\left(P P_{1}\right)^{\prime} P Q_{1} G_{2}^{-1} q, \\
Q x=-\left(Q P_{1} G_{2}^{-1} B+Q Q_{1}\left(P Q_{1}\right)^{\prime}\right) P P_{1} x+Q Q_{1}\left(P Q_{1} G_{2}^{-1} q\right)^{\prime}+Q P_{1} G_{2}^{-1} q \\
\left.-5^{\prime}\right) \quad Q Q_{1}\left(P Q_{1}\right)^{\prime} P Q_{1} G_{2}^{-1} q,
\end{gathered}
$$

and the solution is decomposed as $x=P P_{1} x+P Q_{1} x+Q x$, where the component $P P_{1} x$ solves the inherent regular ODE (2.4); $Q x$ and $P Q_{1} x$ are given by (2.5) and (2.6), respectively (cf. [2]).

In the next section, we will generalize the solvability results given in $[8]$ in the sense that we will do with less smoothness. The new inherent regular ODE, which uses a different projector instead of $P P_{1}$, permits us to obtain new insights into the asymptotic stability behaviour of numerical approximations (Section 3 ).

Note that, by construction, $P(t) P_{1}(t)$ projects onto $P(t) S_{1}(t)$ along $N(t) \oplus N_{1}(t)$, i.e., it is related to the decomposition

$$
\mathbb{R}^{m}=P(t) S_{1}(t) \oplus N(t) \oplus N_{1}(t) .
$$

2.2. Solvability and decoupling. Now we also use the orthoprojector $V(t)$, which projects along $S_{1}(t)$. Then, $I-V(t)$ is the orthoprojector onto $S_{1}(t)$. Because of $N(t) \subset S_{1}(t)$, we have that $V(t) Q(t)=0$, and

$$
\Pi(t):=P(t)(I-V(t))
$$

is a projector, too. Obviously, we have im $\Pi(t)=P(t) S_{1}(t)$. Since the two projectors $\Pi(t)$ and $P(t) P_{1}(t)$ have the same image space $P(t) S_{1}(t)$, it follows that $P P_{1} \Pi=\Pi, \Pi P P_{1}=P P_{1}$.

Additionally, we denote the orthoprojector onto im $A_{1}(t)$ by $I-W(t)$. As we will see below, the projection $W$ extricates exactly what derivative-free part of equations has to be differentiated once when reducing the index. 
The following example of a Hessenberg form DAE is to make the meaning of the different projectors more transparent.

Example. Given a Hessenberg form DAE of size 2 that contains $n_{1}$ equations with derivatives and $n_{2}$ derivative-free ones

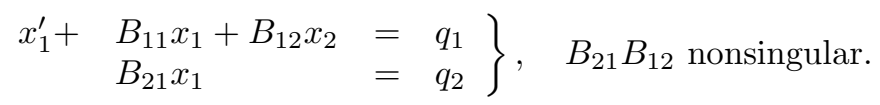

Here we have a constant nullspace $N, N=\left\{z: z_{1}=0\right\}$, and further

$$
\begin{gathered}
Q=\left(\begin{array}{cc}
0 & 0 \\
0 & I
\end{array}\right), \quad A_{1}=\left(\begin{array}{cc}
I & B_{12} \\
0 & 0
\end{array}\right), \quad W=\left(\begin{array}{cc}
0 & 0 \\
0 & I
\end{array}\right) \\
S(t)=S_{1}(t)=\left\{z: B_{21}(t) z_{1}=0\right\}, \quad S(t) \cap N=N, \\
N_{1}(t)=\left\{z: z_{1}+B_{12}(t) z_{2}=0\right\}, \\
N_{1}(t) \cap S_{1}(t)=\left\{z: z_{1}+B_{12}(t) z_{2}=0, B_{21}(t) z_{1}=0\right\}=\{0\}
\end{gathered}
$$

due to the nonsingularity of $B_{21} B_{12}$. The projector $P P_{1}$ is given by

$$
P P_{1}=\left(\begin{array}{cc}
I-L & 0 \\
0 & 0
\end{array}\right),
$$

where $L=B_{12}\left(B_{21} B_{12}\right)^{-1} B_{21}$ is also a projector $\left(L(t)\right.$ projects $\mathbb{R}^{n_{1}}$ onto im $B_{12}(t)$ along ker $\left.B_{21}(t)\right)$. The orthoprojector along $S_{1}(t)$ is now

$$
V(t)=\left(\begin{array}{cc}
B_{21}(t)^{+} B_{21}(t) & 0 \\
0 & 0
\end{array}\right)
$$

and

$$
\begin{aligned}
\Pi(t) & =\left(\begin{array}{cc}
I-B_{21}(t)^{+} B_{21}(t) & 0 \\
0 & 0
\end{array}\right), \\
\operatorname{im~} \Pi(t) & =P(t) S_{1}(t)=\left\{z: B_{21}(t) z_{1}=0, z_{2}=0\right\} \\
& =\operatorname{ker} B_{21}(t) \times\{0\} .
\end{aligned}
$$

It is well known that, in Hessenberg systems, all derivative-free equations should be differentiated when deriving a solution via a reduction step. Consequently, we can put up with $B_{21} \in C^{1}$ (hence, $V, \Pi \in C^{1}$ ) instead of assuming $L \in C^{1}$ (i.e., $\left.P P_{1} \in C^{1}\right)$.

Next, we collect some nice properties of our projectors and matrices to be used below:

1) im $A_{1}=\operatorname{ker} W$ and $\operatorname{im} A_{1}=\operatorname{ker} G_{2} P Q_{1} G_{2}^{-1}$. Hence there are two projectors $W$ and $G_{2} P Q_{1} G_{2}^{-1}$ along im $A_{1}$. Therefore

$$
W=W G_{2} P Q_{1} G_{2}^{-1}, \quad G_{2} P Q_{1} G_{2}^{-1}=G_{2} P Q_{1} G_{2}^{-1} W .
$$

2) From $W B=W G_{2} P Q_{1} G_{2}^{-1} B=W G_{2} P Q_{1} G_{2}^{-1} B P Q_{1}=W B P Q_{1}$ and from $P Q_{1} G_{2}^{-1} W B=P Q_{1}$ it follows that

$$
\operatorname{ker} W B=\operatorname{ker} P Q_{1}=\operatorname{ker} Q_{1}=S_{1}, \quad \operatorname{im} W B=\operatorname{im} W .
$$

3) $V=(W B)^{+} W B, W=\left(I-A_{1} A_{1}^{+}\right)=\left(P Q_{1} G_{2}^{-1}\right)^{+} P Q_{1} G_{2}^{-1}$, and $W B=$ $\left(P Q_{1} G_{2}^{-1}\right)^{+} P Q_{1}$. 
4) $V=V P Q_{1}, P Q_{1}=P Q_{1} V, V=V P, V=V Q_{1}$, and

$$
\begin{aligned}
V G_{2}^{-1} & =(W B)^{+} W B G_{2}^{-1}=(W B)^{+} W B P Q_{1} G_{2}^{-1} \\
& =\left(W B^{+} W G_{2} P Q_{1} G_{2}^{-1} B P Q_{1} G_{2}^{-1}=(W B)^{+} W G_{2} P Q_{1} G_{2}^{-1}=(W B)^{+} W .\right.
\end{aligned}
$$

Due to the second property, $W B \in C^{1}$ implies that both subspaces im $A_{1}$ and $S_{1}$, and also the projector $W$, are continuously differentiable. Namely, $W(t) B(t)$ has constant rank $m-\nu$; thus $(W B)^{+}$belongs to $C^{1}$ at the same time as $W B$ does. Therefore, $V=(W B)^{+} W B$ and $W B(W B)^{+}=W W^{+}=W$ are from $C^{1}$. The subspaces $S_{1}$ and im $A_{1}$ appear to be the nullspaces of the continuously differentiable projectors $V$ and $W$, respectively; hence they are spanned by continuously differentiable base functions.

The third property indicates that, for continuously differentiable $P Q_{1} G_{2}^{-1}$ and $P Q_{1}$, we also have continuously differentiable $W$ and $V$. However, as we may see in the case of the special Hessenberg form DAE above, the opposite is not true. In this sense, Theorem 2.2 below generalizes the related results of 8 .

To get a better insight we reconsider the decoupled form (2.4), (2.5), (2.6) of the DAE (2.1) and try to reformulate it in such a way that (2.4) returns into a regular ODE for the solution component $\Pi x$. Now, we do not assume $P P_{1}, P Q_{1}$ to be from $C^{1}$ as we did in order to obtain (2.4 $)$, (2.5 ), but we need only $P, \Pi$ and $V$ to be continuously differentiable now.

Equation (2.6) yields immediately

$$
V x=(W B)^{+} W q .
$$

To realize what equation (2.4) looks like in more detail, we derive

$$
\begin{aligned}
P P_{1}(P x)^{\prime} & =P P_{1}(\Pi+P V)(P x)^{\prime}=\Pi(P x)^{\prime}+P P_{1} V(P x)^{\prime} \\
& =(\Pi x)^{\prime}-\Pi^{\prime} P x+P P_{1}(V x)^{\prime}-P P_{1} V^{\prime} P x .
\end{aligned}
$$

Consequently, (2.4) can be rewritten as

$$
\begin{gathered}
(\Pi x)^{\prime}-\Pi^{\prime}(\Pi x+P V x)-P P_{1} V^{\prime}(\Pi x+P V x)+P P_{1}(V x)^{\prime} \\
+P P_{1} G_{2}^{-1} B P P_{1}(\Pi x+P V x)=P P_{1} G_{2}^{-1} q .
\end{gathered}
$$

Analogously, with $Q Q_{1}(P x)^{\prime}=Q Q_{1} V(P x)^{\prime}=Q Q_{1}(V x)^{\prime}-Q Q_{1} V^{\prime}(\Pi x+P V x)$, equation (2.5) can be reformulated:

$$
\begin{aligned}
-Q Q_{1}(V x)^{\prime} & +Q Q_{1} V^{\prime}(\Pi x+P V x) \\
& +Q P_{1} G_{2}^{-1} B P P_{1}(\Pi x+P V x)+Q x=Q P_{1} G_{2}^{-1} q .
\end{aligned}
$$

If $x \in C_{N}^{1}$ solves the DAE (2.1), then $(W B)^{+} W q=V x=V P x$ belongs to $C^{1}$ since $V$ and $P x$ do. We are allowed to replace $V x$ and $(V x)^{\prime}$ in (2.9) and (2.10) by means of (2.8). This leads us to consider the ODE

$$
\begin{aligned}
u^{\prime}-\Pi^{\prime} & u-P P_{1} V^{\prime} u+P P_{1} G_{2}^{-1} B P P_{1} u \\
= & \Pi^{\prime} P(W B)^{+} W q+P P_{1} V^{\prime} P(W B)^{+} W q-P P_{1} G_{2}^{-1} B P P_{1}(W B)^{+} W q \\
& -P P_{1}\left((W B)^{+} W q\right)^{\prime}+P P_{1} G_{2}^{-1} q,
\end{aligned}
$$

and to call it an inherent regular ODE. If we multiply equation (2.11) by $(I-\Pi)$, we obtain

$$
(I-\Pi) u^{\prime}-(I-\Pi) \Pi^{\prime} u=0,
$$


hence

$$
((I-\Pi) u)^{\prime}+\Pi^{\prime}(I-\Pi) u=0 .
$$

It follows that if $u \in C^{1}$ solves (2.11), then the function $\omega=(I-\Pi) u$ satisfies the homogeneous regular ODE $\omega^{\prime}+\Pi^{\prime} \omega=0$. If, additionally, $\omega\left(t_{*}\right)=$ $\left(I-\Pi\left(t_{*}\right)\right) u\left(t_{*}\right)=0$, then $\omega(t)$ vanishes identically, i.e., $u(t)=\Pi(t) u(t), t \in J$. This proves the following assertion.

Lemma 2.1. The subspace $\operatorname{im} \Pi(t) \subset \mathbb{R}^{m}$ represents an invariant subspace of the inherent regular ODE (2.11).

As far as the homogeneous case $q=0$ is concerned, we know from (2.8) that each solution of $A x^{\prime}+B x=0$ has a trivial component $V x=0$. Using (2.9), (2.10), and taking into account the inherent regular ODE, we may express each solution as $x=\Pi x+Q x=\Pi_{\text {can }} u$, where $u$ solves (2.11) as well as the initial condition $u\left(t_{o}\right) \in \operatorname{im~} \Pi\left(t_{0}\right)$, and

$$
\Pi_{\text {can }}:=K P P_{1}, K:=\left(I-Q P_{1} G_{2}^{-1} B P P_{1}-Q Q_{1} V^{\prime} P P_{1}\right) .
$$

The matrix function $K$ is nonsingular, thus $\operatorname{ker} \Pi_{\text {can }}(t)=\operatorname{ker} P(t) P_{1}(t), t \in J$. It is easily checked that $\Pi_{\text {can }}$ is also a projector. $\Pi_{\text {can }}$ is said to be the canonical projector for the index-2 case. It projects onto the solution space of the homogeneous DAE along $N \oplus N_{1}$, as we will see below.

Theorem 2.2. Given an index-2 DAE (2.1) with continuously differentiable $W B$ and $q \in\left\{p \in C: W p \in C^{1}\right\}$.

(i) Then, the IVP for (2.1) with the initial condition $\Pi\left(t_{0}\right)\left(x\left(t_{0}\right)-x^{0}\right)=0$, $x^{0} \in \mathbb{R}^{m}$, has exactly one $C_{N}^{1}$-solution.

(ii) Exactly one solution of the homogeneous equation at $t_{0}$ passes through each $x_{0} \in \operatorname{im} \Pi_{\mathrm{can}}\left(t_{0}\right)$.

Proof.

(i) Denote by $u \in C^{1}$ the solution of the regular ODE (2.12) that satisfies the initial condition $u\left(t_{0}\right)=\Pi\left(t_{0}\right) x^{0}$. Then we decompose the function $x$ as

$$
\begin{gathered}
x=u+v+w, \\
v:=(W B)^{+} W q \in C^{1}, \\
w:=Q P_{1} G_{2}^{-1} q+Q Q_{1} v^{\prime}-Q Q_{1} V^{\prime}(u+P v)-Q P_{1} G_{2}^{-1} B P P_{1}(u+P v) .
\end{gathered}
$$

Due to this construction we have $v=V v, w=Q w, P x=u+P v \in C^{1}$, $x \in C, \Pi\left(t_{0}\right) x\left(t_{0}\right)=u\left(t_{0}\right)=\Pi\left(t_{0}\right) x^{0}$. Finally, straightforward checking shows that $x$ indeed satisfies the DAE (2.1).

(ii) Solve the special IVP for $q=0$ and $x^{0}:=x_{0}=\Pi_{\text {can }}\left(t_{0}\right) x_{0}$. Its solution is $x=\Pi_{\mathrm{can}} u$, and we have, in particular,

$$
\begin{aligned}
x\left(t_{0}\right) & =\Pi_{\mathrm{can}}\left(t_{0}\right) u\left(t_{0}\right)=\Pi_{\mathrm{can}}\left(t_{0}\right) \Pi\left(t_{0}\right) x_{0} \\
& =\Pi_{\mathrm{can}}\left(t_{0}\right) \Pi\left(t_{0}\right) \Pi_{\mathrm{can}}\left(t_{0}\right) x_{0}=\Pi_{\mathrm{can}}\left(t_{0}\right) \Pi\left(t_{0}\right)\left(P P_{1}\right)\left(t_{0}\right) x_{0} \\
& =\Pi_{\mathrm{can}}\left(t_{0}\right)\left(P P_{1}\right)\left(t_{0}\right) x_{0} \\
& =\Pi_{\mathrm{can}}\left(t_{0}\right) x_{0}=x_{0} .
\end{aligned}
$$

Corollary. On each compact interval $\left[t_{0}, T\right]$, the perturbation index of an index-2 tractable DAE is two. 
Proof. For each $T>t_{0}$, there is a constant $K_{T}$ such that the estimation

$$
\|x\|_{T} \leq K_{T}\left\{\left|\Pi\left(t_{0}\right) x^{0}\right|+\|q\|_{T}+\left\|(V q)^{\prime}\right\|_{T}\right\}
$$

holds for all IVP solutions with $x^{0} \in \mathbb{R}^{m}, q \in C, V q \in C^{1}$, where $\|y\|_{T}:=$ $\max \left\{|y(t)|: t \in\left[t_{0}, T\right]\right\}$.

Example. For the Hessenberg form DAE

$$
\left.\begin{array}{ccc}
x_{1}^{\prime}+B_{11} x_{1}+B_{12} x_{2} & = & q_{1} \\
B_{21} x_{1} & = & q_{2}
\end{array}\right\}
$$

the conditions of Theorem (2.2) are satisfied if $q_{1} \in C, q_{2} \in C^{1}$, and $B_{21} \in C^{1}$. The initial condition reads, in detail,

$$
0=\Pi\left(t_{0}\right)\left(x\left(t_{0}\right)-x^{0}\right)=\left(\begin{array}{c}
\left(I-B_{21}\left(t_{0}\right)^{+} B_{21}\left(t_{0}\right)\right)\left(x_{1}\left(t_{0}\right)-x_{1}^{0}\right) \\
0
\end{array}\right) .
$$

2.3. Index reduction by differentiation. Considering the DAE (2.1), we observe immediately that each solution has always to proceed in the set

$$
\begin{aligned}
\mathcal{M}(t) & :=\left\{z \in \mathbb{R}^{m}: B(t) z-q(t) \in \operatorname{im} A(t)\right\} \\
& =\left\{z \in \mathbb{R}^{m}:(I-W(t))(B(t) z-q(t)) \in \operatorname{im} A(t), W(t)(B(t) z-q(t))=0\right\},
\end{aligned}
$$

which is called a constraint manifold. Under the conditions of Theorem 2.2, the part of equations described by

$$
W B x=W q
$$

can be differentiated to obtain

$$
W B x^{\prime}+(W B)^{\prime} x=(W q)^{\prime} .
$$

Here, $W B x^{\prime}$ stands for $W B\left\{(P x)^{\prime}-P^{\prime} x\right\}$. On the other hand, due to (2.1) we may express $P x^{\prime}:=(P x)^{\prime}-P^{\prime} x=P A^{+}(q-B x)$. In consequence, the relation

$$
W B A^{+}(q-B x)+W(W B)^{\prime} x=W(W q)^{\prime}
$$

has to be satisfied additionally, i.e., the solution has also to proceed in the so-called hidden constraint manifold

$$
\begin{aligned}
\mathcal{H}(t):= & \left\{z \in \mathbb{R}^{m}:\left(-W(t) B(t) A(t)^{+} B(t)+W(W B)^{\prime}(t)\right) z\right. \\
& \left.=W(W q)^{\prime}(t)-W(t) B(t) A(t)^{+} q(t)\right\} .
\end{aligned}
$$

As we will see below, the set

$$
\mathcal{M}_{1}(t):=\mathcal{M}(t) \cap \mathcal{H}(t)
$$

is characteristic of the DAE (2.1). $\mathcal{M}_{1}$ contains all solutions (for given $q$ ), and it is filled by those solutions. One could call $\mathcal{M}_{1}(t)$ the state manifold. For the case of a homogeneous equation, $S(t)=\mathcal{M}(t)$ is trivially given. Moreover, there is a close relationship between our subspace $S_{1}(t)$ and the state manifold $\mathcal{M}_{1}(t)$.

Lemma 2.3. For a homogeneous index-2 tractable DAE (2.1) with continuously differentiable $W B$, one has

$$
\left.\mathcal{M}_{1}(t)\right|_{q=0}=\left\{z \in S_{1}(t): Q(t) z=-\left(\left(Q P_{1} G_{2}^{-1} B+Q Q_{1} G_{2}^{-1}(W B)^{\prime}\right) P P_{1}\right)(t) z\right\},
$$

and

$$
\left.P(t) \mathcal{M}_{1}(t)\right|_{q=0}=P(t) S_{1}(t)
$$


Proof. The second relation is a simple consequence of a representation of $\mathcal{M}_{1}(t)$, which we want to show now. $z \in \mathcal{M}_{1}$ means, by definition,

$$
-W B A^{+} B z+W(W B)^{\prime} z=0, B z+A w=0 \text { for a certain } w=P w .
$$

This is equivalent to

$$
\begin{gathered}
W B w+W(W B)^{\prime} z=0, w=-P A^{+} B z, \quad P Q_{1} z=0, \\
Q z-Q Q_{1} w+Q P_{1} G_{2}^{-1} B P P_{1} z+Q P_{1} P P^{\prime} Q z=0,
\end{gathered}
$$

and to

$$
\begin{gathered}
P Q_{1} z=0, \quad w=-P A^{+} B z, 0=W B w+W(W B)^{\prime} P P_{1} z+W(W B)^{\prime} Q z \\
=W B w+W(W B)^{\prime} P P_{1} z+W B P^{\prime} Q z, \\
Q z-Q Q_{1} w-Q Q_{1} P^{\prime} Q z+Q P_{1} G_{2}^{-1} B P P_{1} z=0 .
\end{gathered}
$$

Because $Q Q_{1} G_{2}^{-1} W B=Q Q_{1} G_{2}^{-1} B=Q Q_{1}$ and $Q Q_{1} G_{2}^{-1} W=Q Q_{1} G_{2}^{-1}$, we find $z \in \mathcal{M}_{1}$ to be characterized by

$$
\begin{gathered}
P Q_{1} z=0, w=-P A^{+} B z \\
Q Q_{1} w+Q Q_{1} P^{\prime} Q z+Q Q_{1} G_{2}^{-1}(W B)^{\prime} P P_{1} z=0, \\
Q z=Q Q_{1} w+Q Q_{1} P^{\prime} Q z-Q P_{1} G_{2}^{-1} B P P_{1} z \\
=-Q Q_{1} P^{\prime} Q z+Q Q_{1} P^{\prime} Q z-Q Q_{1} G_{2}^{-1}(W B)^{\prime} P P_{1} z-Q P_{1} G_{2}^{-1} B P P_{1} z \\
=-Q Q_{1} G_{2}^{-1}(W B)^{\prime} P P_{1} z-Q P_{1} G_{2}^{-1} B P P_{1} z .
\end{gathered}
$$

Note that the linear space $\left.\mathcal{M}_{1}(t)\right|_{q=0}$ represents the tangent space for $\mathcal{M}_{1}(t)$. Of course, this is much more important for nonlinear problems. Lemma 2.3 shows $P(t) S_{1}(t)$ to be a kind of practical substitute for the tangent space. It should be stressed once more that we are looking for $C_{N}^{1}$ solutions and that there is no natural need for $Q$-components of the elements of the tangent spaces.

Next, rewrite the DAE (2.1) as

$$
A x^{\prime}+(I-W)(B x-q)+W(B x-q)=0
$$

and replace the part $W(B x-q)=0$ by its differentiated form

$$
W\left\{W B x^{\prime}+(W B)^{\prime} x-(W q)^{\prime}\right\}=0,
$$

to compose the new DAE

$$
(A+W B) x^{\prime}+\left((I-W) B+W(W B)^{\prime}\right) x=(I-W) q+W(W q)^{\prime} .
$$

Denote $\tilde{A}:=A+W B, \tilde{B}:=(I-W) B+W(W B)^{\prime}$ and consider (2.14) in more detail. Since $(A(t)+W(t) B(t)) z=0$ decomposes into $A z=0, W B z=0$, we have $\operatorname{ker} \tilde{A}(t) \equiv \operatorname{ker} A(t)=N(t)$. In consequence, the solutions of (2.15) belong to the same class $C_{N}^{1}$ as the solutions of (2.1). Further, we have

$$
\begin{aligned}
& \tilde{S}(t)=\left\{z \in \mathbb{R}^{m}: \tilde{B}(t) z \in \operatorname{im} \tilde{A}(t)\right\} \\
&=\left\{z \in \mathbb{R}^{m}:\right.(I-W(t)) B(t) z \in \operatorname{im} A(t), \\
&\left.W(t)(W B)^{\prime}(t) z=W(t) B(t) A(t)^{+}(I-W(t)) B(t) z\right\},
\end{aligned}
$$


and

$$
\begin{gathered}
\widetilde{\mathcal{M}}(t)=\left\{z \in \mathbb{R}^{m}:(I-W(t)(B(t) z-q(t)) \in \operatorname{im} A(t),\right. \\
\left.W(t)(W B)^{\prime}(t) z-W(t)(W q)^{\prime}(t)=W(t) B(t) A(t)^{+}(I-W(t))(B(t) z-q(t))\right\}, \\
\widetilde{\mathcal{M}}(t) \cap\left\{z \in \mathbb{R}^{m}: W(t)(B(t) z-q(t))=0\right\}=\mathcal{M}_{1}(t) .
\end{gathered}
$$

Theorem 2.4. Given the conditions of Theorem 2.2.

(i) Then equation (2.14) is index-1 tractable.

(ii) Exactly one solution at $t_{*} \in J$ passes through each $x_{*} \in \widetilde{\mathcal{M}}\left(t_{*}\right)$.

(iii) $\mathcal{M}_{1}(t)$ and $\mathcal{M}(t)$ form invariant subsets for (2.14).

\section{Proof.}

(i) We check the nonsingularity of the matrix $\tilde{G}_{1}(t) \in L\left(\mathbb{R}^{m}\right), \tilde{G}_{1}:=A+W B+$ $\left((I-W) B+W(W B)^{\prime}\right) Q \cdot \tilde{G}_{1} z=0$ yields $A z+(I-W) B Q z=0, \quad W B z=$ $-W(W B)^{\prime} Q z=-W B P^{\prime} Q z$, hence $P Q_{1} z=-P Q_{1} P^{\prime} Q z,(A+B Q) z=0$. Because $(A+B Q)\left(I-P P^{\prime} Q\right)=A_{1}$ and $A_{1}\left(I+P P^{\prime} Q\right)=A+B Q$, the element $\left(I+P P^{\prime} Q\right) z=\tilde{z}$ belongs to ker $A_{1}=N_{1}$, i.e., $\tilde{z}=Q_{1} \tilde{z}$. On the other hand, $P Q_{1} z+P Q_{1} P^{\prime} Q z=0$ implies $P Q_{1}\left(I+P P^{\prime} Q\right) z=0$, i.e., $P Q_{1} \tilde{z}=0$ and finally $\tilde{z}=0, z=0$.

(ii) Due to the index-1 property, this assertion is now given by the index-1 results, e.g., in [2].

(iii) Let $x$ denote a solution of the index-1 tractable DAE (2.14) and $x\left(t_{*}\right) \in$ $\mathcal{M}\left(t_{*}\right)$. Then, 2.14 gives

$$
A x^{\prime}+(I-W) B x=(I-W) q, \quad W B x^{\prime}+W(W B)^{\prime} x=W(W q)^{\prime} .
$$

Consider the function $\alpha:=W(B x-q)$. Derive

$$
\begin{aligned}
\alpha^{\prime} & =(W B)^{\prime} x+W B x^{\prime}-(W q)^{\prime} \\
& =(W B)^{\prime} x-W(W B)^{\prime} x+W(W q)^{\prime}-(W q)^{\prime} \\
& =(I-W)(W B)^{\prime} x-(I-W)(W q)^{\prime} \\
& =-(I-W)^{\prime}(W B x-W q)=W^{\prime} \alpha
\end{aligned}
$$

Since $x\left(t_{*}\right) \in \mathcal{M}\left(t_{*}\right)$ implies $\alpha\left(t_{*}\right)=W\left(t_{*}\right)\left(B\left(t_{*}\right) x\left(t_{*}\right)-q\left(t_{*}\right)\right)=0$, the function $\alpha$ vanishes identically; hence $A x^{\prime}+B x=q$ is satisfied. Therefore, $x_{*}(t) \in \mathcal{M}\left(t_{*}\right)$ implies $x(t) \in \mathcal{M}(t)$, but also $x(t) \in \mathcal{M}_{1}(t)$ for all $t \in J$.

Corollary. The index-2 tractable DAE has differentiation index two.

Example. For the Hessenberg system

$$
\left.\begin{array}{rl}
x_{1}^{\prime}+B_{11} x_{1}+B_{12} x_{2} & =q_{1} \\
B_{21} x_{1} & =q_{2}
\end{array}\right\}
$$


we have now

$$
\begin{aligned}
\mathcal{M}(t)= & \left\{z: B_{21}(t) z_{1}-q_{2}(t)=0\right\} \\
\mathcal{H}(t)= & \left\{z:-B_{21}(t) B_{11}(t) z_{1}-B_{21}(t) B_{12}(t) z_{2}\right. \\
& \left.\quad+B_{21}^{\prime}(t) z_{1}=q_{2}^{\prime}(t)-B_{21}(t) q_{1}(t)\right\} \\
\mathcal{M}_{1}(t)=\{z: & B_{21}(t) z_{1}=q_{2}(t),\left(B_{21}(t) B_{12}(t)\right) z_{2} \\
& \left.=B_{21}^{\prime}(t) z_{1}-B_{21}(t) B_{11}(t) z_{1}-q_{2}^{\prime}(t)+B_{21}(t) q_{1}(t)\right\}, \\
\tilde{\mathcal{M}}(t)=\{z: & \left.B_{21}^{\prime}(t) z_{1}-q_{2}^{\prime}(t)=B_{21}(t)\left(B_{11}(t) z_{1}+B_{12}(t) z_{2}-q_{1}(t)\right)\right\}, \\
\tilde{\mathcal{M}}(t) \cap\{z: & \left.B_{21}(t) z_{1}=q_{2}(t)\right\}=\mathcal{M}_{1}(t) .
\end{aligned}
$$

The index-reduced equation (2.15) is, as expected,

$$
\left.\begin{array}{r}
x_{1}^{\prime}+B_{11} x_{1}+B_{12} x_{2}=q_{1} \\
B_{21} x_{1}^{\prime}+B_{21}^{\prime} x_{1}=q_{2}^{\prime}
\end{array}\right\} .
$$

2.4. Contractivity. Now we turn to the asymptotic behaviour of the solutions of the homogeneous equations

$$
A x^{\prime}+B x=0 .
$$

As we know, the solutions may be represented by

$$
x=K P P_{1} u=\Pi_{\mathrm{can}} u,
$$

where $u$ solves the inherent regular ODE

$$
\begin{gathered}
u^{\prime}=\Pi^{\prime} u+P P_{1} V^{\prime} u-P P_{1} G_{2}^{-1} B P P_{1} u, \\
u\left(t_{0}\right) \in \operatorname{im} \Pi\left(t_{0}\right) .
\end{gathered}
$$

Recall once more that $\operatorname{im} \Pi(t)$ is an invariant subspace of the ODE (2.16), i.e.,

$$
u(t)=\Pi(t) u(t), t \in J .
$$

Obviously, the stability behaviour of $x$ is mainly governed by the dynamics of the inherent regular ODE, that is, by $u$. Hence, it would be nice to formulate criteria on how the flow of a regular ODE behaves within a given invariant subspace.

The matrix coefficient of the inherent regular ODE (2.16) has to be expected to be singular. In particular, for constant subspaces $N$ and $S_{1}$, it is simply the matrix $P P_{1} G_{2}^{-1} B P P_{1}$, which has at least the nullspace $N \oplus N_{1}$ of dimension $m-r+\nu \geq$ 2. The standard approaches to estimate how the solutions grow usually relate to all solutions of (2.16), included those solutions that do not start in im $\Pi\left(t_{0}\right)$. No exponential decay can be realized in this way. The standard techniques are somewhat coarse. Hence, we try to improve them by relating all things to the invariant subspaces.

For a given subspace $U \subset \mathbb{R}^{m}$ we introduce the matrix semi-norm

$$
\|G\|^{U}:=\max \left\{\frac{|G z|}{|z|}: z \neq 0, z \in U\right\}
$$

so that $|G z| \leq\|G\|^{U}|z|$ for all $z \in U$.

Then, we define the logarithmic matrix norm relative to the subspace $U$ by

$$
\mu^{U}(G):=\lim _{h \rightarrow 0} \frac{1}{h}\left(\|I+h G\|^{U}-1\right) .
$$


This logarithmic norm relative to $U$ is well defined and has similar properties as the standard version of Dahlquist for $U=\mathbb{R}^{m}$ (cf. [9]). This generalization is in fact very natural and straightforward. On the other hand, it is worth mentioning that $\mu^{U}(G)$ is a special case of the logarithmic norm proposed in [10] for matrix pencils.

Lemma 2.5. Given a regular linear $O D E$

$$
u^{\prime}(t)+M(t) u(t)=0, t \in J=\left[t_{0}, \infty\right),
$$

which has the invariant subspace $U(t) \subset \mathbb{R}^{m}, t \in J$. Let the function

$$
\gamma(t):=\int_{t_{0}}^{t} \mu^{U(s)}(-M(s)) d s, t \in J,
$$

be well defined, i.e., the integrals do exist. Then, for all ODE solutions starting with initial values $u\left(t_{0}\right) \in U\left(t_{0}\right)$, the inequality

$$
|u(t)| \leq e^{\gamma(t)}\left|u\left(t_{0}\right)\right|, t \geq t_{0},
$$

holds.

Proof. This proof follows the lines of the standard theory 9]. Given a solution with $u\left(t_{0}\right) \in U\left(t_{0}\right)$, we have $u(t) \in U(t)$, for all $t \in\left[t_{0}, \infty\right)$. Denote $m(t):=|u(t)|, t \in$ $\left[t_{0}, \infty\right)$, and derive that

$$
\begin{aligned}
m(t+h) & =\left|u(t)+h u^{\prime}(t)+o(h)\right|=|(I-h M(t)) u(t)+o(h)| \\
& \leq \|\left(I-h M(t) \|^{U(t)}|u(t)|+o(h) .\right.
\end{aligned}
$$

Thus

$$
\frac{1}{h}(m(t+h)-m(t)) \leq \frac{1}{h}\left(\|I-h M(t)\|^{U(t)}-1\right) m(t)-o\left(h^{0}\right) .
$$

Letting $h \rightarrow 0$, we obtain

$$
D_{+} m(t) \leq \mu^{U(t)}(-M(t)) m(t), t \in\left[t_{0}, \infty\right),
$$

where $D_{+} m(t)$ denotes the respective Dini derivative. By Peano's Lemma it follows immediately that

$$
m(t) \leq e^{\gamma(t)} m\left(t_{0}\right)
$$

Theorem 2.6. Let (2.15) be index-2 tractable with continuously differentiable $W B$. Then, the estimate

$$
|x(t)| \leq\left\|\Pi_{\text {can }}(t)\right\| e^{\gamma(t)}\left|\Pi\left(t_{0}\right) x\left(t_{0}\right)\right|, t \geq t_{0},
$$

with $\gamma(t):=\int_{t_{0}}^{t} \mu^{\left(P S_{1}\right)(\tau)}(-M(\tau)) d \tau, t \geq t_{0}$, holds for each solution, provided that $\gamma(t)$ is well defined. Here,

$$
M:=-\Pi^{\prime}-P P_{1} V^{\prime}+P P_{1} G_{2}^{-1} B P P_{1}
$$

is the coefficient matrix of the inherent regular ODE (2.16). 
Proof. Applying Lemma 2.5 to (2.16), which has the invariant subspace im $\Pi(t)=$ $P(t) S_{1}(t)$, yields $|u(t)| \leq e^{\gamma(t)}\left|u\left(t_{0}\right)\right|, t \geq t_{0}$, provided that $u\left(t_{0}\right) \in \operatorname{im} \Pi\left(t_{0}\right)$. The DAE solutions have the representation $x(t)=\Pi_{\text {can }}(t) u(t)$, and $u\left(t_{0}\right)=\Pi\left(t_{0}\right) x\left(t_{0}\right)$.

Corollary. If there is a $\beta>0$ such that $\mu^{\left(P S_{1}\right)(t)}(-M(t)) \leq-\beta, t \geq t_{0}$, all DAE solutions satisfy the inequality

$$
|x(t)| \leq\left\|\Pi_{\text {can }}(t)\right\| e^{-\beta\left(t-t_{0}\right)} \mid \Pi\left(t_{0}\right) x\left(t_{0} \mid, t \geq t_{0} .\right.
$$

Hence, if $\Pi_{\text {can }}(t)$ grows "moderately" like polynomials or is bounded by $\left\|\Pi_{\mathrm{can}}(t)\right\| \leq$ $C e^{\alpha t}, t \geq t_{0}$, with $\alpha<\beta$, then the solutions decrease exponentially.

To realize whether numerical approximations reflect the stability behaviour of the true solution well, one often uses the notion of contractivity and so-called onesided Lipschitz conditions (cf. [9]). This approach applies also to DAEs provided that we relate the things again to our invariant subspaces.

Standard numerical integration methods applied to index-2 DAE are expected to work well if the basic nullspace $N$ is constant. However, it is well known that these methods may fail in case this nullspace rotates with time. This is why we suppose $P^{\prime}=0$ now.

If the vector norm used to define the logarithmic norm is related to an inner product, then

$$
\langle G z, z\rangle \leq \mu^{U}(G)|z|^{2} \quad \text { for all } z \in U \text {. }
$$

Definition. An index-2 tractable DAE with constant $P$ is said to be contractive if there are an inner product $\langle$,$\rangle and a constant \beta>0$ such that the inequality

$$
\langle y, P x\rangle \leq-\beta|P x|^{2}
$$

is valid for all $y, x \in \mathbb{R}^{m}, t \in\left[t_{0}, \infty\right)$, with

$$
A(t) y+B(t) x=0, \quad Q y=0, \quad V(t) y=V(t) \Pi^{\prime}(t) x .
$$

This definition is given in [6] for the case of a continuously differentiable $Q_{1}$ and a real scalar product. If $Q_{1} \in C^{1}$, we may make use of

$$
Q_{1}(t) y=Q_{1}(t) V(t) y=Q_{1}(t) \Pi^{\prime}(t) x=-Q_{1}^{\prime}(t) \Pi(t) x=-Q_{1}^{\prime}(t) P P_{1}(t) x
$$

and arrive at the same expression as used in [6] instead of (2.21). By decoupling $A(t) y+B(t) x=0$, we find that (2.21) leads to

$$
y+M(t) \Pi(t) x=0, V(t) x=0, P x=\Pi(t) x .
$$

Therefore, 2.20) then reads

$$
\langle-M(t) \Pi(t) x, \Pi(t) x\rangle \leq-\beta|\Pi(t) x|^{2},
$$

which means a contractivity condition for the inherent regular ODE relative to the invariant subspace $\operatorname{im} \Pi(t)=P S_{1}(t)$. 


\section{ANALYZING NUMERICAL INTEGRATION METHOdS FOR LINEAR INDEX-2 DAES}

In this section we derive conditions for preserving stability properties of numerical methods when solving index-2 linear DAEs. Let the conditions of Theorem 2.2 be fulfilled in the following. As in [6] we assume $N(t)$ to be constant; otherwise it is well known that those methods will fail. We shall consider the discrete version of the decoupling of the last section for two of the most important families of methods. An analogous analysis was made in [6] using the standard approach mentioned in subsection 2.1. In that paper they obtained as a sufficient condition for preserving the stability properties that both subspaces $N_{1}$ and $S_{1}$ have to be time invariant. By the discrete decoupling with the new approach described in subsection 2.2, we shall get a weaker condition than in [6]. Namely, the time invariance of $S_{1}$ will do.

3.1. BDF. Consider the homogeneous system

$$
A(t) x^{\prime}(t)+B(t) x(t)=0, \quad t \in J:=\left[t_{0}, \infty\right) .
$$

For homogeneous index-2 tractable DAEs (3.1), the solutions are given by the expression

$$
x=\left(I-Q P_{1} G_{2}^{-1} B P P_{1}-Q Q_{1} V^{\prime} P P_{1}\right) P P_{1} u=\Pi_{\text {can }} u,
$$

where $u$ solves the regular ODE

$$
u^{\prime}-\Pi^{\prime} u-P P_{1} V^{\prime} u+P P_{1} G_{2}^{-1} B P P_{1} u=0,
$$

with $u\left(t_{0}\right) \in \operatorname{Im} \Pi\left(t_{0}\right)$. Suppose $P^{\prime}=0$.

If the projector $V$ is time invariant, or equivalently, $\Pi=P(I-V)$ is so, this solution representation simplifies to

$$
\begin{gathered}
x=\left(I-Q P_{1} G_{2}^{-1} B P P_{1}\right) P P_{1} u, \\
u^{\prime}+P P_{1} G_{2}^{-1} B P P_{1} u=0 .
\end{gathered}
$$

Given the step size $h>0, t_{i}=t_{0}+i h, i \in \mathbb{N}$, the BDF applied to (3.1) reads

$$
A_{i} \sum_{j=0}^{k} \alpha_{j} x_{i-j}+h B_{i} x_{i}=0, \quad i \geq k,
$$

where the starting values $x_{0}, \ldots, x_{k-1}$ are assumed to be given.

First, we multiply by $\left((W B)^{+} W\right)_{i}$ and obtain

$$
V_{i} x_{i}=0, \quad i \geq k,
$$

which means that this component is correctly computed. Let us now consider what happens with the other components. Assuming that the previous values $x_{i-1}, \ldots, x_{i-k}$ as well as $x_{i}$ also fulfill (3.7), we have $x_{i}=\Pi_{i} x_{i}+Q x_{i}, \forall i$, and, keeping in mind that $P^{\prime}=0$, we can rewrite 3.6 as

$$
A_{i} \sum_{j=0}^{k} \alpha_{j}(\Pi x)_{i-j}+h B_{i}(\Pi x)_{i}+h B_{i}(Q x)_{i}=0, \quad i \geq k .
$$

Multiplying by $G_{2, i}^{-1}$, we obtain

$$
P_{1, i} P \sum_{j=0}^{k} \alpha_{j}(\Pi x)_{i-j}+h\left(G_{2}^{-1} B P P_{1}\right)_{i}(\Pi x)_{i}+h Q x_{i}=0 .
$$


For notational brevity, let $H:=P P_{1} G_{2}^{-1} B P P_{1}$ and $u_{j}=\Pi_{j} x_{j}$. Multiplying (B.8) by $P P_{1, i}$ yields

$$
P P_{1, i} \sum_{j=0}^{k} \alpha_{j} u_{i-j}+h H_{i} u_{i}=0
$$

which is equivalent to

$$
\begin{gathered}
\sum_{j=0}^{k} \alpha_{j} u_{i-j}+h H_{i} u_{i}+P P_{1, i} \sum_{j=0}^{k} \alpha_{j} u_{i-j}-\sum_{j=0}^{k} \alpha_{j} u_{i-j}=0 \\
\sum_{j=0}^{k} \alpha_{j} u_{i-j}+h H_{i} u_{i}+\sum_{j=1}^{k} \alpha_{j}\left(\Pi_{i}-\Pi_{i-j}\right) u_{i-j}+\sum_{j=1}^{k} \alpha_{j}\left[P P_{1, i}-\Pi_{i}\right] u_{i-j}=0
\end{gathered}
$$

$$
\sum_{j=0}^{k} \alpha_{j} u_{i-j}+h H_{i} u_{i}+\sum_{j=1}^{k} \alpha_{j}\left(\Pi_{i}-\Pi_{i-j}\right) u_{i-j}+P P_{1, i} \sum_{j=1}^{k} \alpha_{j}\left(V_{i}-V_{i-j}\right) u_{i-j}=0 .
$$

This is the discrete analogue of (3.3).

For the $Q$-component we multiply (3.8) by $Q$, thus obtaining

$$
\begin{gathered}
h Q x_{i}-Q Q_{1, i} \sum_{j=0}^{k} \alpha_{j} u_{i-j}+h\left(Q P_{1} G_{2}^{-1} B P P_{1}\right)_{i} u_{i}=0, \\
Q x_{i}=Q Q_{1, i} \frac{1}{h} \sum_{j=0}^{k} \alpha_{j} u_{i-j}-\left(Q P_{1} G_{2}^{-1} B P P_{1}\right)_{i} u_{i} .
\end{gathered}
$$

Now, regarding (3.9) and (3.1), we observe that, if the projection $\Pi$ is constant, then the BDF discretization of (3.1) coincides with the corresponding method applied to (3.5) and formula (3.4). Namely, we have $x_{i}=\Pi_{\text {can }}\left(t_{i}\right) \Pi_{i} x_{i}=\Pi_{\text {can }}\left(t_{i}\right) u_{i}$.

Theorem 3.1. Let (3.1) be index-2 tractable with continuously differentiable WB and $P^{\prime}=0$. Suppose the starting values satisfy $x_{i} \in \mathcal{M}\left(t_{i}\right), i=0, \ldots, k-1$. Then the BDF (3.6) applied to (3.1) generates exactly the same BDF method applied to the inherent $O D E(3.3)$ if the projection $V$ (the subspace $S_{1}$ ) is constant.

Remarks. 1) The numerical approximation $x_{i}=\Pi_{\text {can }}\left(t_{i}\right) u_{i}$ reflects the asymptotic behaviour of the true solution $x\left(t_{i}\right)=\Pi_{\mathrm{can}}\left(t_{i}\right) u\left(t_{i}\right)$ nicely if the BDF works well for the inherent regular ODE (3.3).

2) Let $S_{1}(t)$ be time-invariant. Then, applying the BDF to (3.1) and decoupling this is exactly the same as decoupling (3.1) first and then applying the BDF to the inherent regular ODE. In this sense, the BDF-discretization and the decoupling commute.

3) In circuit simulation, the DAEs obtained by the classical modified nodal analysis fulfil the condition $V^{\prime}=0$ (cf. [7]).

In the previous analysis we have checked whether the BDF scheme is transmitted to the inherent regular ODE (3.3). Analogously, we could also check if the BDF method is transmitted to the reduced index equation (2.14). This would be very helpful, because in the constant null space case we know that the BDF preserves its asymptotic stability properties (cf. [4]). 
Looking at (3.6), we immediately see that $x_{i} \in \mathcal{M}\left(t_{i}\right)$. Splitting (3.6) with the projections $W_{i}$ and $I-W_{i}$ yields, $\forall i \geq k$,

$$
\begin{gathered}
A_{i} \sum_{j=0}^{k} \alpha_{j} x_{i-j}+h\left(I-W_{i}\right)(B x)_{i}=0, \\
(W B x)_{i}=0 .
\end{gathered}
$$

However, in general we cannot hope that the approximation $x_{i}$ satisfies the hidden constraint. In fact, this will be a key condition for the BDF methods to preserve their asymptotic stability properties. To see this we rewrite (3.11) as

$$
(A+W B)_{i} \sum_{j=0}^{k} \alpha_{j} x_{i-j}+h\left(I-W_{i}\right)(B x)_{i}-(W B)_{i} \sum_{j=0}^{k} \alpha_{j} x_{i-j}=0 .
$$

Here we see that, if we had the relation

$$
h W_{i}(W B)_{i}^{\prime} x_{i}=-(W B)_{i} \sum_{j=0}^{k} \alpha_{j} x_{i-j}
$$

then we would really obtain the BDF discretization of the index-1 problem (2.14). The above condition can also be written as

$$
W_{i}\left\{(W B)_{i} \frac{1}{h} \sum_{j=0}^{k} \alpha_{j} x_{i-j}+(W B)_{i}^{\prime} x_{i}\right\}=0,
$$

which is nothing but the BDF discretization of the differentiated constraint. Furthermore, (3.6) provides

$$
P \frac{1}{h} \sum_{j=0}^{k} \alpha_{j} x_{i-j}=-P\left(A^{+} B x\right)_{i}
$$

so (3.13) can also be written as

$$
W_{i}\left\{-(W B)_{i}\left(A^{+} B x\right)_{i}+(W B)_{i}^{\prime} x_{i}\right\}=0 \text {, i.e., } x_{i} \in \mathcal{H}\left(t_{i}\right) .
$$

Theorem 3.2. Let (3.1) be index-2 tractable with continuously differentiable $W B$ and $P^{\prime}=0$. Then a $B D F$ method applied to (3.1) generates exactly the same BDF method applied to the index-1 DAE (2.14) if the condition (3.13) is fulfilled.

Remarks. 1) The projection $\left(I-Q \widetilde{A}_{1}^{-1} \widetilde{B}\right) P$ plays the role of the canonical projector for the index-1 tractable DAE; the solution of (2.14) satisfies $x=$ $\left(I-Q \widetilde{A}_{1}^{-1} \widetilde{B}\right) P x$.

2) Condition (3.13) means that the numerical approximation for $x$ must lie on the hidden constraint manifold in every integration step. This is more difficult to check than the condition $V^{\prime}=0$. The BDF solution of (3.1) at the point $t_{i}$ is $x_{i}=(\Pi x)_{i}+Q x_{i}$. Inserting this expression in the left side of (3.13), we obtain

$$
W_{i}\left\{(W B)_{i} \frac{1}{h} \sum_{j=0}^{k} \alpha_{j}(\Pi x+Q x)_{i-j}+(W B)_{i}^{\prime}(\Pi x+Q x)_{i}\right\} .
$$

Then, assuming $V$ to be constant and taking into account that $W B \Pi=$ $W B Q=0,(W B)^{\prime} \Pi=(W B \Pi)^{\prime}=0,(W B)^{\prime} Q=(W B Q)^{\prime}=0$, one concludes that (B.13) is indeed fulfilled. 
3.2. Runge-Kutta methods. The implicit Runge-Kutta methods can be realized for (3.1) in the following way [11]. Given an approximation $x_{l-1}$ of the solution at $t_{l-1}$, a new approximation $x_{l}$ at $t_{l}=t_{l-1}+h$ is obtained from

$$
x_{l}=x_{l-1}+h \sum_{i=1}^{s} b_{i} X_{l i}^{\prime},
$$

where the $X_{l i}^{\prime}$ are defined by

$$
\begin{gathered}
A_{l i} X_{l i}^{\prime}+B_{l i} X_{l i}=0, i=1, \ldots, s, \\
t_{l i}=t_{l-1}+c_{i} h,
\end{gathered}
$$

and the internal stages are given by

$$
X_{l i}=x_{l-1}+h \sum_{j=1}^{s} a_{i j} X_{l j}^{\prime}, i=1, \ldots, s .
$$

The coefficients $a_{i j}, b_{i}, c_{i}$ determine the IRK method, and $s$ is the number of stages. We define the matrix $\mathcal{A}:=\left(a_{i j}\right)_{i, j=1}^{s}$ and the vectors $b:=\left(b_{1}, \ldots, b_{s}\right)^{T}$, $c:=\left(c_{1}, \ldots, c_{s}\right)^{T}$. A condition for $X_{l 1}^{\prime}, \ldots, X_{l s}^{\prime}$ to be uniquely defined by (3.15)(3.16) is the non-singularity of $\mathcal{A}$, [12], which we shall assume in the following. Denoting $\widehat{\mathcal{A}}:=\mathcal{A}^{-1}=\left(\hat{a}_{i j}\right)_{i, j=1}^{s}$ and $\rho:=1-\sum_{i=1}^{s} \sum_{j=1}^{s} b_{i} \hat{a}_{i j}$, we see that (3.14)(3.16) is equivalent to

$$
\begin{gathered}
x_{l}=\rho x_{l-1}+\sum_{i=1}^{s} \sum_{j=1}^{s} b_{i} \hat{a}_{i j} X_{l j}, \\
A_{l i} \sum_{j=1}^{s} \hat{a}_{i j}\left(X_{l j}-x_{l-1}\right)+h B_{l i} X_{l i}=0, i=1, \ldots, s .
\end{gathered}
$$

Looking at (3.18) we observe that the internal stages do not depend on $Q x_{l-1}$. Further, $X_{l j} \in \mathcal{M}\left(t_{l j}\right)$.

The special class of IRK methods (IRK(DAE) [4]) with coefficients

$$
b_{i}=a_{s i}, \quad i=1, \ldots, s, \quad c_{s}=1,
$$

is known to stand out from all IRK methods in view of its applicability to DAEs. Since $\rho=0$ in this case, the new value $x_{l}=X_{l s}$ always belongs to the constraint manifold $\mathcal{M}\left(t_{l}\right)$.

For index-2 Hessenberg equations, the fact that $x_{l} \in \mathcal{M}\left(t_{l}\right)$ simplifies to

$$
B_{21} x_{1, l}=q_{2, l} .
$$

In general, if (3.19) is not fulfilled, then we have $\rho \neq 0$, and $x_{l}$ does not belong to $\mathcal{M}\left(t_{l}\right)$ any more. Since this behaviour is a source of instability (for $h \rightarrow 0$ ), Ascher and Petzold 13 propose another version for the application of IRK methods to index-2 Hessenberg systems (cf. the discussion of Hessenberg systems in subsection 2.2 ), the so-called Projected IRK methods (PIRK). Actually, after realizing the standard internal stage computation, the recursion (3.17) for the Hessenberg system (cf. subsection 2.2) is now replaced by

$$
\hat{x}_{1, l}=\rho \hat{x}_{1, l-1}+\sum_{i=1}^{s} \sum_{j=1}^{s} b_{i} \hat{a}_{i j} \widehat{X}_{1, l j}+B_{12}\left(t_{l}\right) \lambda_{l},
$$


and $\lambda_{l}$ is determined by

$$
B_{21}\left(t_{l}\right) \hat{x}_{1, l}=q_{2, l} .
$$

If we multiply (3.20) by $I-L_{l}$ ( $L$ is defined in subsection 2.2), $\lambda_{l}$ can be eliminated:

$$
\left(I-L_{l}\right) \hat{x}_{1, l}=\rho\left(I-L_{l}\right) \hat{x}_{1, l-1}+\sum_{i=1}^{s} \sum_{j=1}^{s} b_{i} \hat{a}_{i j}\left(I-L_{l}\right) \widehat{X}_{1, l j} .
$$

On the other hand, 3.21) is equivalent to

$$
L_{l} \hat{x}_{1, l}=B_{12}\left(t_{l}\right)\left(B_{21}\left(t_{l}\right) B_{12}\left(t_{l}\right)\right)^{-1} q_{2, l} .
$$

It should be mentioned that, for IRK(DAE), the projected version is exactly the same as the original one, since (3.19) implies $\lambda_{l}=0$ in (3.20), (3.21). An immediate generalization of PIRK methods to fully implicit linear index-2 systems is suggested in [6]:

$$
\begin{gathered}
P P_{1, l} \hat{x}_{l}=\rho P P_{1, l} \hat{x}_{l-1}+\sum_{i=1}^{s} \sum_{j=1}^{s} b_{i} \hat{a}_{i j} P P_{1, l} \widehat{X}_{l j}, \\
Q_{1, l} \hat{x}_{l}=Q_{1, l} A_{2, l}^{-1} q_{l} .
\end{gathered}
$$

Since the internal stages do not depend on $Q \hat{x}_{l-1}$, there is no need to compute $Q \hat{x}_{l}$ at this place.

Now return to the standard IRK methods (3.17)-(3.18) for a homogeneous equation. First, we multiply (3.18) by $\left((W B)^{+} W\right)_{l i}$, and obtain for all internal stages

$$
(V X)_{l i}=0, \quad i=1, \ldots, s,
$$

which means that the $V$-component is correctly computed for all internal stages. This is a consequence of the fact that $X_{l i} \in \mathcal{M}\left(t_{l i}\right)$. Next, multiplying (3.17) by $V_{l}$ results in

$$
\begin{gathered}
(V x)_{l}=\rho V_{l} x_{l-1}+\sum_{i=1}^{s} \sum_{j=1}^{s} b_{i} \hat{a}_{i j} V_{l} X_{l j}, \\
(V x)_{l}=\rho(V x)_{l-1}+\rho\left(V_{l}-V_{l-1}\right) x_{l-1}+\sum_{i=1}^{s} \sum_{j=1}^{s} b_{i} \hat{a}_{i j}\left(V_{l}-V_{l j}\right) X_{l j},
\end{gathered}
$$

which shows that an IRK scheme does not generate an approximation $x_{l}$ in $\mathcal{M}\left(t_{l}\right)$ in general. Let us see what happens with the П-component. Multiplying (3.17) by $\Pi_{l}$, we obtain

$$
\begin{gathered}
(\Pi x)_{l}=\rho \Pi_{l} x_{l-1}+\sum_{i=1}^{s} \sum_{j=1}^{s} b_{i} \hat{a}_{i j} \Pi_{l} X_{l j}, \\
(\Pi x)_{l}=\rho(\Pi x)_{l-1}+\sum_{i=1}^{s} \sum_{j=1}^{s} b_{i} \hat{a}_{i j}(\Pi X)_{l j}+\rho\left(\Pi_{l}-\Pi_{l-1}\right) x_{l-1} \\
+\sum_{i=1}^{s} \sum_{j=1}^{s} b_{i} \hat{a}_{i j}\left(\Pi_{l}-\Pi_{l j}\right) X_{l j},
\end{gathered}
$$


and for (3.18), assuming that $x_{l-1} \in \mathcal{M}\left(t_{l-1}\right)$, we can write

$$
A_{l i} \sum_{j=1}^{s} \hat{a}_{i j}\left[(\Pi X)_{l j}-(\Pi x)_{l-1}\right]+h(B X)_{l i}=0 .
$$

Multiplying by $G_{2, l i}^{-1}$ and using the shorter notation $u_{j}:=\Pi_{j} x_{j}, U_{l j}:=\Pi_{l j} X_{l j}$, we obtain

$$
P_{1, l i} P \sum_{j=1}^{s} \hat{a}_{i j}\left[U_{l j}-u_{l-1}\right]+h\left(G_{2}^{-1} B U\right)_{l i}+h Q X_{l i}=0 .
$$

Then, multiplying by $P P_{1, l i}$ leads to

$$
\begin{gathered}
P P_{1, l i} \sum_{j=1}^{s} \hat{a}_{i j}\left[U_{l j}-u_{l-1}\right]+h H_{l i} U_{l i}=0 \\
\sum_{j=1}^{s} \hat{a}_{i j}\left[U_{l j}-u_{l-1}\right]+h H_{l i} U_{l i}-\sum_{j=1}^{s} \hat{a}_{i j}\left[U_{l j}-u_{l-1}\right] \\
+P P_{1, l i} \sum_{j=1}^{s} \hat{a}_{i j}\left[U_{l j}-u_{l-1}\right]=0, \\
\sum_{j=1}^{s} \hat{a}_{i j}\left[U_{l j}-u_{l-1}\right]-\sum_{j=1}^{s} \hat{a}_{i j}\left(\Pi_{l j}-\Pi_{l i}\right) U_{l j}+\sum_{j=1}^{s} \hat{a}_{i j}\left[P P_{1, l i}-\Pi_{l i}\right] U_{l j} \\
+h H_{l i} U_{l i}+\sum_{j=1}^{s} \hat{a}_{i j} P Q_{1, l i} u_{l-1}=0, \\
\sum_{j=1}^{s} \hat{a}_{i j}\left[U_{l j}-u_{l-1}\right]+h H_{l i} U_{l i}-\sum_{j=1}^{s} \hat{a}_{i j}\left(\Pi_{l j}-\Pi_{l i}\right) U_{l j}+\sum_{j=1}^{s} \hat{a}_{i j} P P_{1, l i} V_{l i} U_{l j} \\
+\sum_{j=1}^{s} \hat{a}_{i j} P Q_{1, l i} u_{l-1}=0, \\
\sum_{j=1}^{s} \hat{a}_{i j}\left[U_{l j}-u_{l-1}\right]+h H_{l i} U_{l i}-\sum_{j=1}^{s} \hat{a}_{i j}\left(\Pi_{l j}-\Pi_{l i}\right) U_{l j}-P P_{1, l i} \sum_{j=1}^{s} \hat{a}_{i j}\left(V_{l j}-V_{l i}\right) U_{l j} \\
+\sum_{j=1}^{s} \hat{a}_{i j} P Q_{1, l i}\left[\Pi_{l-1}-\Pi_{l i}\right] u_{l-1}=0 .
\end{gathered}
$$

Finally, for the $Q$-component we multiply (3.17) by $Q$ and obtain

$$
Q x_{l}=\rho Q x_{l-1}+\sum_{i=1}^{s} \sum_{j=1}^{s} b_{i} \hat{a}_{i j} Q X_{l j},
$$

and (3.29) multiplied by $Q$ gives

$$
\begin{aligned}
& -Q Q_{1, l i} \sum_{j=1}^{s} \hat{a}_{i j}\left[U_{l j}-u_{l-1}\right]+h\left(Q P_{1} G_{2}^{-1} B U\right)_{l i}+h Q X_{l i}=0, \\
& Q X_{l i}=Q Q_{1, l i} \frac{1}{h} \sum_{j=1}^{s} \hat{a}_{i j}\left[U_{l j}-u_{l-1}\right]-\left(Q P_{1} G_{2}^{-1} B U\right)_{l i} .
\end{aligned}
$$


After these algebraic manipulations we can extract the desired conclusions. If $S_{1}(t)$ is time-invariant $\left(V^{\prime}=0\right)$, then (3.27), (3.26), (3.28), (3.30), (3.31) and (3.32) can be reduced to

$$
\begin{gathered}
(V x)_{l}=\rho(V x)_{l-1}, \\
(\Pi x)_{l}=\rho(\Pi x)_{l-1}+\sum_{i=1}^{s} \sum_{j=1}^{s} b_{i} \hat{a}_{i j}(\Pi X)_{l j}, \\
(Q x)_{l}=\rho(Q x)_{l-1}+\sum_{i=1}^{s} \sum_{j=1}^{s} b_{i} \hat{a}_{i j}(Q X)_{l j}, \\
(V X)_{l i}=0, \quad i=1, \ldots, s \\
\sum_{j=1}^{s} \hat{a}_{i j}\left[(\Pi X)_{l j}-(\Pi x)_{l-1}\right]+h H_{l i}(\Pi X)_{l i}=0, \quad i=1, \ldots, s
\end{gathered}
$$

and

$$
(Q X)_{l i}=-\left(Q P_{1} G_{2}^{-1} B \Pi X\right)_{l i}, \quad i=1, \ldots, s
$$

In (3.33) we have left the term $\rho(P V x)_{l-1}$ to emphasize that an "undesirable" recursion occurs in the $P V$-component. An even worse situation occurs in the $Q$ component: here the expression (3.35) does not correspond to (3.4) any more, which may cause instabilities in the computation. What really is true is the following:

Theorem 3.3. Suppose (3.1) to be index-2 tractable with continuous differentiable $W B$ and $P^{\prime}=0$. Then, an IRK method applied to (3.1) generates exactly the same IRK method applied to the inherent ODE (3.3) if the projection $V$ (the subspace $\left.S_{1}\right)$ is constant and the initial value $x_{0}$ belongs to $\mathcal{M}\left(t_{0}\right)$.

Here, the IRK(DAE) methods show their good properties, $\rho=0$ in this case, and

$$
(V x)_{l}=(V X)_{l s}=0, \quad(Q x)_{l}=(Q X)_{l s}=-\left(Q P_{1} G_{2}^{-1} B \Pi X\right)_{l s} ;
$$

thus, there is no recursion, neither in the $P V$-component nor in the $Q$-component, and these components are computed correctly. Hence, for this type of method we can state:

Theorem 3.4. For a constant projection $V$, an IRK(DAE) method applied to the index-2 tractable DAE (3.1) with a continuously differentiable $W B$ and $P^{\prime}=0$ yields $x_{l}=\Pi_{\text {can }}\left(t_{l}\right) u_{l}, x_{l} \in \mathcal{M}_{1}\left(t_{l}\right)$.

Next, let us briefly come back to the PIRK methods (3.24), (3.25). Our decoupling for the internal stages $X_{l i}$ holds true also in this case for the $\widehat{X}_{l i}$ values. For a homogeneous system, under the assumptions of Theorem 3.3 we obtain

$$
\begin{array}{r}
P P_{1, l} \hat{x}_{l}=\rho P P_{1, l} \hat{x}_{l-1}+\sum_{i=1}^{s} \sum_{j=1}^{s} b_{i} \hat{a}_{i j} P P_{1, l} \widehat{X}_{l j}, \\
Q_{1, l} \hat{x}_{l}=0 .
\end{array}
$$

The second equation is equivalent to

$$
V \hat{x}_{l}=0 .
$$


Inserting $\hat{x}_{l}=\Pi \hat{x}_{l}+Q \hat{x}_{l}, \hat{x}_{l-1}=\Pi \hat{x}_{l-1}+Q \hat{x}_{l-1}$ and $\widehat{X}_{l j}=\Pi \widehat{X}_{l, j}+Q \widehat{X}_{l, j}$ in the equation for the $P P_{1}$-component and taking into account that $\Pi$ and $Q$ are constant provides

$$
(\Pi \hat{x})_{l}=\rho(\Pi \hat{x})_{l-1}+\sum_{i=1}^{s} \sum_{j=1}^{s} b_{i} \hat{a}_{i j}(\Pi \hat{X})_{l j},
$$

which is identical with (3.34). Hence, the application of a PIRK scheme solves the problem with the "undesirable" iteration in the $V\left(Q_{1}\right)$-component, but, as the $Q$ component is the same as in a "standard" IRK method, we still have the recursion (3.35) for this component. Consequently, a result like Theorem 3.4 is not possible, but the analogue of Theorem 3.3 remains true for PIRK methods.

In the same way as it was done for BDF methods, we shall analyze IRK methods by means of the index reduction technique.

Considering again the IRK scheme (3.17), (3.18), we split (3.18) with the projections $I-W_{l i}$ and $W_{l i}$ and obtain

$$
\begin{aligned}
& A_{l i} \sum_{j=1}^{s} \hat{a}_{i j}\left(X_{l j}-x_{l-1}\right)+h\left(I-W_{l i}\right) B_{l i} X_{l i}=0, i=1, \ldots, s, \\
& (W B X)_{l i}=0, i=1, \ldots, s .
\end{aligned}
$$

However, the hidden restriction need not be fulfilled in general, neither for the stages $X_{l i}$ nor for $x_{l}$.

Transforming (3.41) appropriately, we obtain

$$
\begin{aligned}
(A+(W B))_{l i} \sum_{j=1}^{s} \hat{a}_{i j}\left(X_{l j}-x_{l-1}\right) & +h\left(I-W_{l i}\right)(B X)_{l i} \\
& -(W B)_{l i} \sum_{j=1}^{s} \hat{a}_{i j}\left(X_{l j}-x_{l-1}\right)=0 .
\end{aligned}
$$

If we had the relation

$$
h\left(W(W B)^{\prime} X\right)_{l i}=-(W B)_{l i} \sum_{j=1}^{s} \hat{a}_{i j}\left(X_{l j}-x_{l-1}\right), \quad i=1, \ldots, s,
$$

then we would arrive at the same method applied to the index-1 problem (2.14) with constant null space $N$. In other words, similarly to the BDF-methods, we obtain

$$
W_{l i}\left\{(W B)_{l i} \frac{1}{h} \sum_{j=1}^{s} \hat{a}_{i j}\left(X_{l j}-x_{l-1}\right)+(W B)_{l i}^{\prime} X_{l i}\right\}=0,
$$

as condition that all stages must fulfil the discretization of the differentiated constraint.

In [4] it is shown that an IRK (DAE) applied to an index-1 DAE with constant leading nullspace generates the same values as the application of the same IRK to the inherent regular ODE, and $x_{l} \in \widetilde{\mathcal{M}}\left(t_{l}\right)$.

Theorem 3.5. Let (3.1) be index-2 tractable with continuously differentiable $W B$ and $P^{\prime}=0$. Then, an IRK(DAE) method applied to (3.1) generates exactly the same IRK for the index-1 DAE (2.14) if condition (3.43) is fulfilled. 
In the same way as we did for the BDF methods, we can show that (3.43) is fulfilled if $V^{\prime}=0$ and $x_{l-1} \in \mathcal{M}\left(t_{l-1}\right)$.

In subsection 2.4 we have extended the notion of contractivity to linear index-2 tractable DAEs. The reason for this concept in regular ODE theory is to generalize the A-stability notion related to the test equation

$$
x^{\prime}=\lambda x
$$

by that of B-stability for a contractive equation (cf. 9]). An assertion of the type "algebraically stable Runge-Kutta methods are B-stable", was shown to be true in [4] for index-1 tractable DAEs provided that (i) the null space $N(t)$ is time-invariant, and (ii) the Runge-Kutta method is an IRK(DAE). In 6] such an assertion was proved for linear index-2 tractable DAEs under the additional conditions that $Q_{1}$ be differentiable, $P P_{1}^{\prime}=0$ and $\left\|\Pi_{\text {can }}(t)\right\| \leq K$, on $\left[t_{0}, \infty\right)$.

Definition. The one-step method $x_{j+1}=\phi\left(x_{j}, t_{j}, h_{j}\right)$ is called B-stable if, for each contractive DAE, the inequalities

$$
\left|P x_{j+1}^{(1)}-P x_{j+1}^{(2)}\right|_{s} \leq\left|P x_{j}^{(1)}-P x_{j}^{(2)}\right|_{s}
$$

and

$$
\left|Q x_{j+1}^{(1)}-Q x_{j+1}^{(2)}\right|_{s} \leq K\left|P x_{j+1}^{(1)}-P x_{j+1}^{(2)}\right|_{s}, \quad j \geq 0,
$$

are satisfied. Here, $K>0$ is a constant, $x_{0}^{(1)}, x_{0}^{(2)}$ are arbitrary consistent initial values and $|\cdot|_{s}$ denotes a suitable norm.

Following the same technique as in [6], we can improve the result of that work by using the projectors $W$ and $V$ instead of $Q_{1} G_{2}^{-1}$ and $Q_{1}$.

Theorem 3.6. Let (3.1) be index-2 tractable with continuously differentiable $W B$; $P^{\prime}=0, V^{\prime}=0$ and $\left\|\Pi_{\text {can }}(t)\right\|$ bounded on $\left[t_{0}, \infty\right)$. Then, each algebraically stable IRK(DAE) applied to (B.1) is B-stable.

\section{Two ILlustrative EXAMPLES}

In this section we would like to illustrate our theory by simple and transparent academic examples.

Example 4.1. The first example is taken from [6],

$$
\left(\begin{array}{c}
x_{1}^{\prime} \\
x_{2}^{\prime} \\
0
\end{array}\right)+\left(\begin{array}{ccc}
\lambda & -1 & -1 \\
\eta t(1-\eta t)-\eta & \lambda & -\eta t \\
1-\eta t & 1 & 0
\end{array}\right)\left(\begin{array}{l}
x_{1} \\
x_{2} \\
x_{3}
\end{array}\right)=0, t \geq 0 .
$$

It is a Hessenberg system with $B_{21} B_{12}=1$ and, thus, index-2 tractable for all $t$. The general exact solution is given by

$$
\begin{aligned}
& x_{1}(t)=x_{1}(0) e^{-\lambda t}, \\
& x_{2}(t)=(\eta t-1) x_{1}(t), \\
& x_{3}(t)=-(\eta t-1) x_{1}(t),
\end{aligned}
$$

which, evidently, is exponentially asymptotically stable for $\lambda>0$.

If we use

$$
P=\left(\begin{array}{lll}
1 & 0 & 0 \\
0 & 1 & 0 \\
0 & 0 & 0
\end{array}\right)
$$


then we get

$$
A_{1}(t)=\left(\begin{array}{ccc}
1 & 0 & -1 \\
0 & 1 & -\eta t \\
0 & 0 & 0
\end{array}\right)
$$

and

$$
S_{1}(t)=\left\{z \in \mathbb{R}^{3}:(1-\eta t) z_{1}+z_{2}=0\right\} .
$$

After computing the canonical projection $Q_{1}$, the projection onto $\operatorname{Ker}\left(A_{1}\right)$ along $S_{1}$, we have

$$
P_{1}(t)=\left(\begin{array}{ccc}
\eta t & -1 & 0 \\
\eta t(\eta t-1) & 1-\eta t & 0 \\
\eta t-1 & -1 & 1
\end{array}\right), \quad P P_{1}(t)=\left(\begin{array}{ccc}
\eta t & -1 & 0 \\
\eta t(\eta t-1) & 1-\eta t & 0 \\
0 & 0 & 0
\end{array}\right)
$$

In [6], as already mentioned, the authors showed their stability results under the condition $P P_{1}^{\prime}=0$, and by means of this example they illustrated that, if this condition fails, even when using methods with good stability properties, the numerical discretizations may show an asymptotic behaviour that is different from that of the exact solution. Note that in this example we have $P P_{1}^{\prime}=0$ for $\eta=0$ only. One integration step with the implicit Euler method consists in solving the following linear system:

$$
\left(\begin{array}{ccc}
1+h \lambda & -h & -h \\
h\left(\eta t_{i+1}\left(1-\eta t_{i+1}\right)-\eta\right) & 1+h \lambda & -h \eta t_{i+1} \\
1-\eta t_{i+1} & 1 & 0
\end{array}\right)\left(\begin{array}{c}
x_{1, i+1} \\
x_{2, i+1} \\
x_{3, i+1}
\end{array}\right)=\left(\begin{array}{c}
x_{1, i} \\
x_{2, i} \\
0
\end{array}\right),
$$

which is invertible for $1+h(\lambda+\eta) \neq 0$. The third equation yields

$$
x_{2, i+1}=\left(\eta t_{i+1}-1\right) x_{1, i+1},
$$

and then, solving the linear system, we obtain the expression

$$
x_{1, i+1}=\frac{1+h \eta}{1+h(\lambda+\eta)} x_{1, i}
$$

for $x_{1, i+1}$.

In order to have a decaying sequence for $x_{1}$, the following condition is needed:

$$
\left|\frac{1+h \eta}{1+h(\lambda+\eta)}\right|<1
$$

This can be violated for $\eta<0$, while the exact solution decays for all $\lambda>0$. We have computed the numerical solution with this method and stepsize $h=0.1$ on $[0,10]$. Figure 1 shows, in a logarithmic scale, the absolute value of the first component at $t=10$ for different values of $\lambda$ and $\eta$.

As predicted by the above analysis, we see that for negative values of $\eta$, if $\lambda$ is not big enough, the numerical approximation "explodes". The gap about $\eta=-10$ is due to the fact that for this value of $\eta$ and $h=10^{-1}$ we have $x_{1, i+1}=0$ for all $i$. Now let us have a look at the projection $V$. According to the expression for $P_{1}$ we deduce that $S_{1}(t)$ is generated by the last two columns of this projector matrix; thus $S_{1}(t)$ and, consequently, also $V(t)$ are independent of $t$ in case of $\eta=0$ only. 


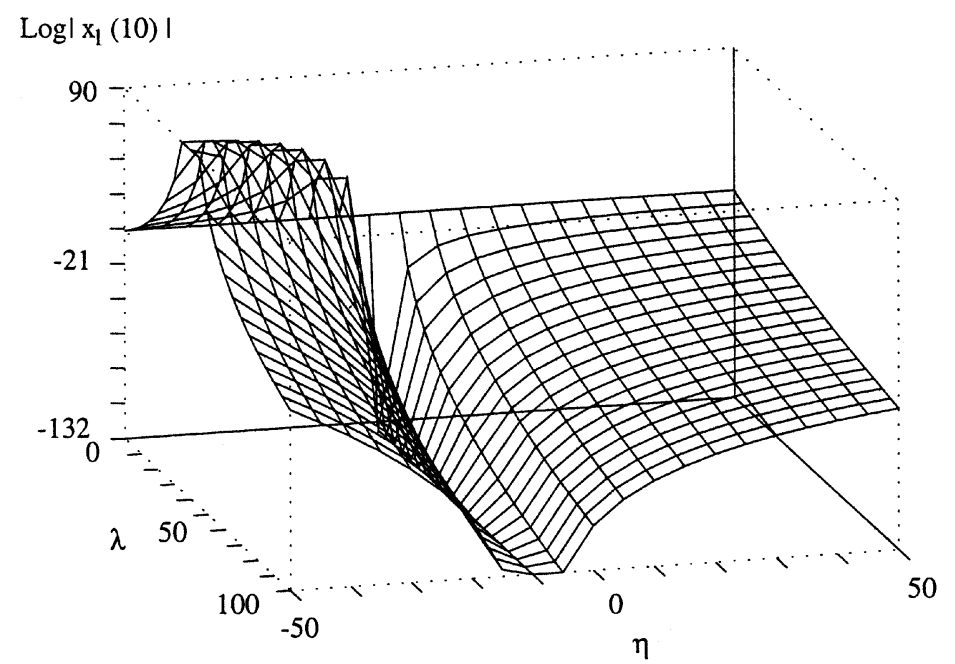

FiguRE 1.

Note that for this example

$$
W=\left(\begin{array}{ccc}
0 & 0 & 0 \\
0 & 0 & 0 \\
0 & 0 & 1
\end{array}\right), \quad(W B)(t)=\left(\begin{array}{ccc}
0 & 0 & 0 \\
0 & 0 & 0 \\
1-\eta t & 1 & 0
\end{array}\right)
$$

hence, for $V$ we obtain the expression

$$
V(t)=\frac{1}{1+(1-\eta t)^{2}}\left(\begin{array}{ccc}
(1-\eta t)^{2} & 1-\eta t & 0 \\
1-\eta t & 1 & 0 \\
0 & 0 & 0
\end{array}\right) .
$$

We compute the matrix $M:=P P_{1} G_{2}^{-1} B-\Pi^{\prime}-P P_{1} V^{\prime}$ of the inherent ODE (3.3), thus obtaining

$$
M(t)=\left(\begin{array}{ccc}
\eta(\eta+\lambda) t-\frac{\eta}{\eta t(\eta t-2)+2} & -(\lambda+\eta)+\frac{\eta(1-\eta t)}{\eta t(\eta t-2)+2} & 0 \\
\frac{\eta t(-2 \lambda+\eta(-3+(\lambda+\eta) t(4+\eta t(\eta t-3))))}{\eta t(\eta t-2)+2} & \lambda-\eta(\lambda+\eta) t+\frac{\eta(2-\eta t)}{\eta t(\eta t-2)+2} & 0 \\
0 & 0 & 0
\end{array}\right) .
$$

Moreover,

$$
\operatorname{Im}(\Pi)=\operatorname{span}\{\mathcal{U}\}, \quad \mathcal{U}:=\left(\begin{array}{c}
1 \\
\eta t-1 \\
0
\end{array}\right) .
$$

In subsection 2.4, motivated by the notion of the logarithmic norm of a matrix corresponding to a matrix semi-norm, we have introduced a contractivity notion for linear index-2 tractable DAEs. We can compute the Euclidean logarithmic norm for this problem in the following way:

$$
\mu_{2}^{\operatorname{Im}(\Pi)}[-M]=\sup _{\substack{x \neq 0 \\ x \in \operatorname{Im}(\Pi)}} \frac{\langle x,-M x\rangle}{\langle x, x\rangle}=\sup _{u_{1} \neq 0} \frac{\left\langle\mathcal{U} u_{1},-M \mathcal{U} u_{1}\right\rangle}{\left\langle\mathcal{U} u_{1}, \mathcal{U} u_{1}\right\rangle},
$$


which simplifies to

$$
\sup _{u_{1} \neq 0} \frac{-\left(\eta+2 \lambda-2 \lambda \eta t+\eta^{2} t(\lambda t-1)\right) u_{1}^{2}}{\left(1+(\eta t-1)^{2}\right) u_{1}^{2}}=-\lambda+\frac{\eta(\eta t-1)}{1+(\eta t-1)^{2}}
$$

and this expression is bounded by $-\lambda+\frac{|\eta|}{2}$. So we have contractivity at least for $\frac{|\eta|}{2}<\lambda$ in the Euclidean norm. Moreover, we can observe that

$$
\lim _{t \rightarrow \infty} \mu_{2}^{\operatorname{Im}(\Pi)}[-M](t)=-\lambda .
$$

Example 4.2. A contrasting example is the following:

$$
\left(\begin{array}{c}
x_{1}^{\prime} \\
x_{2}^{\prime} \\
0
\end{array}\right)+\left(\begin{array}{ccc}
\lambda & (\eta t-1)^{2} & -(\eta t-1) \\
\eta t(\eta t-1) & \lambda & -\eta t \\
1 & -1 & 0
\end{array}\right)\left(\begin{array}{l}
x_{1} \\
x_{2} \\
x_{3}
\end{array}\right)=0, t \geq 0
$$

whose exact solution for $x_{1}(0)=1$ is

$$
\begin{aligned}
& x_{1}(t)=e^{-\lambda t}, \\
& x_{2}(t)=x_{1}(t), \\
& x_{3}(t)=(\eta t-1) x_{1}(t),
\end{aligned}
$$

with exponential asymptotic stability for $\lambda>0$.

Let us compute the relevant matrices and subspaces. Taking, also in this case, $P$ as in the first example, we obtain, for $A_{1}$ and the canonical projector $P_{1}$,

$$
A_{1}(t)=\left(\begin{array}{ccc}
1 & 0 & 1-\eta t \\
0 & 1 & -\eta t \\
0 & 0 & 0
\end{array}\right), \quad P_{1}(t)=\left(\begin{array}{ccc}
\eta t & 1-\eta t & 0 \\
\eta t & 1-\eta t & 0 \\
1 & -1 & 1
\end{array}\right) .
$$

Thus, $P P_{1}$ is again not constant, but if we look at $S_{1}$ we see that

$$
S_{1}=\left\{z \in \mathbb{R}^{3}: z_{1}=z_{2}\right\}=\operatorname{span}\left\{\left(\begin{array}{l}
1 \\
1 \\
0
\end{array}\right),\left(\begin{array}{l}
0 \\
0 \\
1
\end{array}\right)\right\},
$$

which is time-independent and so is $V$, too. As in Example 4.1 the projector $W$ is given by

$$
W=\left(\begin{array}{lll}
0 & 0 & 0 \\
0 & 0 & 0 \\
0 & 0 & 1
\end{array}\right)
$$

and

$$
W B=\left(\begin{array}{rrr}
0 & 0 & 0 \\
0 & 0 & 0 \\
1 & -1 & 0
\end{array}\right)
$$

A computation of $V$ gives

$$
V=\frac{1}{2}\left(\begin{array}{rrr}
1 & -1 & 0 \\
-1 & 1 & 0 \\
0 & 0 & 0
\end{array}\right)
$$




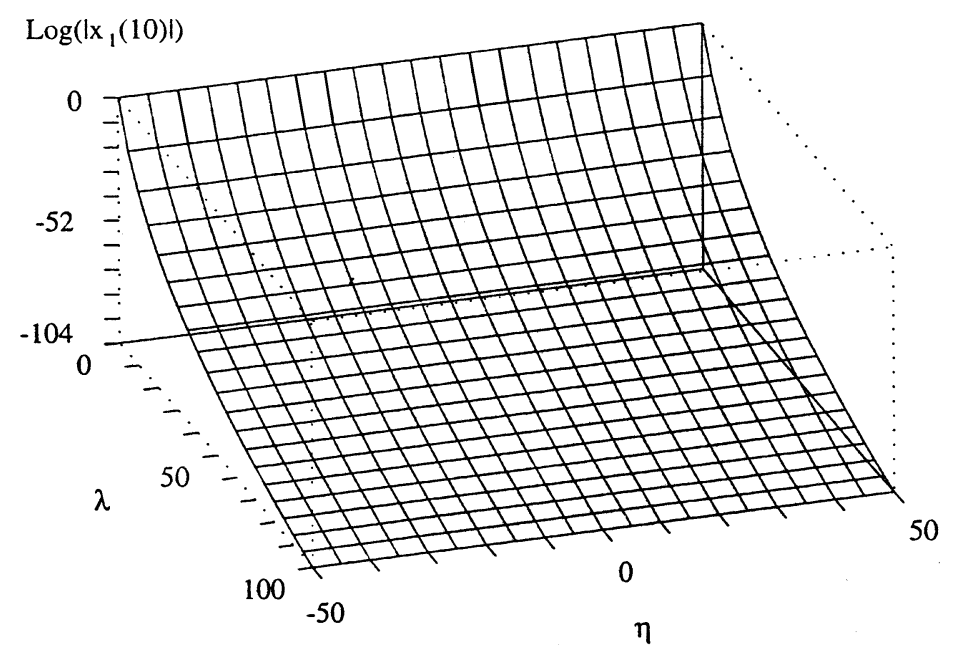

FIGURE 2.

Hence, the assumptions of Theorem 3.4 are fulfilled for the implicit Euler method. An integration step of this method

$$
\left(\begin{array}{ccc}
1+h \lambda & h\left(\eta t_{i}-1\right)^{2} & -h\left(\eta t_{i}-1\right) \\
h \eta t_{i}\left(\eta t_{i}-1\right) & 1+h \lambda & -h \eta t_{i} \\
1 & -1 & 0
\end{array}\right)\left(\begin{array}{l}
x_{1, i} \\
x_{2, i} \\
x_{3, i}
\end{array}\right)=\left(\begin{array}{c}
x_{1, i-1} \\
x_{2, i-1} \\
0
\end{array}\right)
$$

provides

$$
x_{1, i}=\frac{1}{1+h \lambda} x_{1, i-1} .
$$

For $\lambda>0$ this fulfils the condition

$$
\left|\frac{1}{1+h \lambda}\right|<1 .
$$

So, in this case the numerical method reflects the asymptotic behaviour of the exact solution. As in the first example, Figure 2 shows the absolute value of $x_{1}$ at $t=10$, for different values of $\lambda$ and $\eta$ in a logarithmic scale using the implicit Euler scheme. Again, we have chosen $h=0.1$.

The coefficient matrix of the inherent ODE (2.9) now reads

$$
M(t)=\left(P P_{1} G_{2}^{-1} B\right)(t)=\left(\begin{array}{ccc}
\eta \lambda t & \lambda(1-\eta t) & 0 \\
\eta \lambda t & \lambda(1-\eta t) & 0 \\
0 & 0 & 0
\end{array}\right) .
$$

Then, taking into account that

$$
\Pi=\frac{1}{2}\left(\begin{array}{ccc}
1 & 1 & 0 \\
1 & 1 & 0 \\
0 & 0 & 0
\end{array}\right), \quad \Pi x=: u=\left(\begin{array}{c}
u_{1} \\
u_{1} \\
0
\end{array}\right),
$$

we can in essence simplify equation (2.9) to

$$
u_{1}^{\prime}+\lambda u_{1}=0 .
$$


On the other hand, if we compute the Euclidean logarithmic norm of $-M$ we obtain

$$
\mu_{2}^{\operatorname{Im}(\Pi)}[-M]=\sup _{\substack{x \neq 0 \\ x \in \operatorname{Im}(\Pi)}} \frac{\langle x,-M x\rangle}{\langle x, x\rangle}=\sup _{u_{1} \neq 0} \frac{\left\langle\mathcal{U} u_{1},-M \mathcal{U} u_{1}\right\rangle}{\left\langle\mathcal{U} u_{1}, \mathcal{U} u_{1}\right\rangle}=-\lambda,
$$

in this example.

\section{REFERENCES}

1. K. E. Brenan, S. L. Campbell and L. R. Petzold: Numerical Solution of InitialValue Problems in Differential-Algebraic Equations. North-Holland (Amsterdam) 1989. MR 92e:65001

2. R. MÄRZ: Numerical methods for differential-algebraic equations. Acta Numerica 1992, 141198. MR 93e:65096

3. E. Hairer AND G. WANner: Solving Ordinary Differential Equations. I, 2nd Edition, Springer, Berlin 1993. MR 94c:65005

4. E. Griepentrog And R. MÄrz: Differential-Algebraic Equations and Their Numerical Treatment. Teubner-Texte zur Mathematik 88, Leipzig 1986. MR 88e:65105

5. M. Hanke AND R. MÄrZ: On the asymptotics in the case of differential-algebraic equations. Talk given in Oberwolfach, October 1995.

6. M. Hanke, E. Izquierdo Macana And R. März: On asymptotics in case of linear index2 differential-algebraic equations. SIAM J. Numer. Anal. 35 (4), 1326-1346, 1998. MR 99d:34003

7. D. Estévez Schwarz And C. Tischendorf: Structural analysis for electric circuits and consequences for MNA. Intern. J. of Circuit Theory and Applications 18, 131-162, 2000.

8. R. MäRZ: Index-2 Differential-Algebraic Equations. Results in Mathematics 15, 149-171, 1989. MR 90a:34008

9. K. DekKer And J. G. Verwer: Stability of Runge-Kutta methods for stiff nonlinear differential equations. CWI Monographs 2, Centre for Mathematics and Computer Science, NorthHolland, 1984. MR 86g:65003

10. I. Higueras and B. Garcia-Celayeta: Logarithmic norms for matrix pencils. SiAM J. Matrix Anal. and Appl. 20 (3), 646-666, 1999. MR 2000c:15014

11. L. R. Petzold: Order results for implicit Runge-Kutta methods applied to differentialalgebraic systems. SIAM J. Numer. Anal. 23, 837-852, 1986. MR 87k:65103

12. E. IzQuierdo MaCANa: Numerische Approximation von Algebro-Differentialgleichungen mit Index 2 mittels impliziter Runge-Kutta-Verfahren. Doctoral thesis, Humboldt-Univ., Fachbereich Mathematik, Berlin, 1993.

13. U. Ascher AND L. R. Petzold: Projected implicit Runge-Kutta methods for differentialalgebraic equations. SIAM J. Numer. Anal. 28, 1097-1120, 1991. MR 92f:65082

Humboldt-University Berlin, Institute of Mathematics, Unter den Linden 6, D-10099 Berlin, Germany

E-mail address: maerz@mathematik.hu-berlin.de

Dresearch Digital Media Systems, Otto-Schimgral-Str. 3, D-10319 Berlin, Germany

E-mail address: rodriguez@dresearch.de 